\title{
1 Dectin-1 mediated DC-SIGN Recruitment to Candida albicans Contact
}

\section{Sites}

3 Authors:

4 Rohan P. Choraghe ${ }^{1}$ and Aaron K. Neumann ${ }^{1,2}$

5 Affiliation:

6 1. Department of Pathology, University of New Mexico, School of Medicine,

7 Albuquerque, NM, USA, 87131

$8 \quad$ 2. Corresponding author: akneumann@salud.unm.edu

9 Keywords:

10 Dectin-1/ß-Glucan/DC-SIGN/mannan/Candida albicans/host-pathogen contact

11 Abstract

12 At host-pathogen contact sites with Candida albicans, Dectin-1 activates pro-

13 inflammatory signaling, while DC-SIGN promotes adhesion to the fungal surface. We

14 observed that Dectin-1 and DC-SIGN collaborate to enhance capture/retention of $C$.

15 albicans under fluid shear culture conditions. Therefore, we devised a cellular model

16 system wherein we could investigate the interaction between these two receptors during

17 the earliest stages of host-pathogen interaction. In cells expressing both receptors, DC-

18 SIGN was quickly recruited to contact sites (103.15\% increase) but Dectin-1 did not

19 similarly accumulate. Once inside the contact site, FRAP studies revealed a strong

20 reduction in lateral mobility of DC-SIGN (but not Dectin-1), consistent with DC-SIGN

21 engaging in multivalent adhesive binding interactions with cell wall mannoprotein ligands.

22 Interestingly, in the absence of Dectin-1 co-expression, DC-SIGN recruitment to the

23 contact was much poorer-only $35.04 \%$. These data suggested that Dectin-1 promotes the

24 active recruitment of DC-SIGN to the contact site. We proposed that Dectin-1 signaling 
25 activates the RHOA pathway, leading to actomyosin contractility that promotes DC-SIGN

26 recruitment, perhaps via the formation of a centripetal ActoMyosin Flow (AMF) directed

27 into the contact site. Indeed, RHOA pathway inhibitors significantly reduced Dectin-1

28 associated DC-SIGN recruitment to the contact site. We used agent based modeling to

29 predict DC-SIGN transport kinetics with ("Directed+Brownian") and without

30 ("Brownian") the hypothesized actomyosin flow-mediated transport. The

31 Directed+Brownian transport model predicted a DC-SIGN contact site recruitment

$32(108.72 \%)$, similar to that we observed experimentally under receptor co-expression.

33 Brownian diffusive transport alone predicted contact site DC-SIGN recruitment of only

$3454.02 \%$. However, this value was similar to experimentally observed recruitment in cells

35 without Dectin-1 or treated with RHOA inhibitor, suggesting that it accurately predicted

36 DC-SIGN recruitment when a contact site AMF would not be generated. TIRF microscopy

37 of nascent cell contacts on glucan-coated glass revealed Dectin-1 dependent DC-SIGN and

38 F-actin (LifeAct) recruitment kinetics to early-stage contact site membranes. DC-SIGN

39 entry followed F-actin with a temporal lag of $8.35 \pm 4.57$ seconds, but this correlation was

40 disrupted by treatment with RHOA inhibitor. Thus, computational and experimental

41 evidence provides support for the existence of a Dectin-1/RHOA-dependent AMF that

42 produces a force to drive DC-SIGN recruitment to pathogen contact sites, resulting in

43 improved pathogen capture and retention by immunocytes. These data suggest that the

44 rapid collaborative response of Dectin-1 and DC-SIGN in early contact sties might be

45 important for the efficient acquisition of yeast under flow conditions, such as those that

46 prevail in circulation or mucocutaneous sites of infection. 


\section{Introduction}

49 C-type lectin receptors play an important role in recognition of the two major fungal

50 cell wall polysaccharide ligands exposed at the host-pathogen interface. DC-SIGN

51 recognizes abundantly exposed N-mannan in the outer cell wall whereas Dectin-1

52 recognizes nanoscale exposures of $\beta$-(1,3)-glucan. Recognition of $\beta$-glucan by Dectin-1

53 contributes to phagocytosis, oxidative burst, regulation of transcription, production of

54 inflammatory cytokines and chemokines, and initiation of adaptive immunity[1]. DC-

55 SIGN is known to mediate intercellular adhesion, as well as antigen uptake and signaling

56 in dendritic cells (DCs)[2]. We are examining the relationship between Dectin-1 and DC-

57 SIGN to understand, in a simplified model, how an effective host-pathogen contact is built.

58 We focused on the earliest events in fungal contact site biogenesis.

59 Initial pathogen capture and formation of a stable contact site are the earliest events

60 that must occur for signaling through antifungal receptors to initiate. Our previous work

61 with zymosan particles demonstrated that human monocyte derived dendritic cells (DC)

62 form durable contacts between the DC plasma membrane and extracellular fungal particles,

63 which may be important for the antigen gathering functions of these cells [3]. Rapid

64 formation of adhesive contact site structures is especially important for C. albicans capture

65 under conditions involving fluid shear stress, for example by reticuloendothelial

66 macrophages capturing yeast in the bloodstream. Fungal recognition under fluid shear also

67 pertains to phagocytes interacting with Candida in the oropharyngeal cavity, a major site

68 of mucocutaneous candidiasis, where the host-pathogen interaction is subject to salivary

69 flow. 
Various authors have described the accumulation of pattern recognition receptors,

71 such as Dectin-1 and DC-SIGN, at fungal contact sites[4-6]. Immune cells must mobilize

72 receptors to these contact sites for activation, crosstalk and amplification of signaling that

73 directs downstream immune responses. In fact, these contact sites achieve an ordered

74 segregation of molecular components with a peripheral zone enriched in the large

75 transmembrane phosphatase CD45 and a central zone where DC-SIGN and Dectin-1

76 concentrates. Such "phagocytic synapses" can also involve the development of barriers to

77 molecular diffusion that support specialized signaling processes occurring therein[7,8].

78 These findings suggest that PRRs are recruited to fungal contacts in some fashion to

79 support their enrichment at these sites. Active and passive transport processes might

80 conceivably account for observed receptor recruitment, but the molecular mechanisms of

81 innate immunoreceptor recruitment in contact sites with C. albicans have not been defined.

82 Previous studies from our group and others have shown the enrichment of DC-

83 SIGN and CD-206 at fungal contact sites[4-6,9]. These studies are typically conducted at

84 longer time scales of hours, which is relevant to processes such as cytokine response and

85 cytotoxic effector responses. However, there is much less information on the dynamics of

86 pattern recognition receptors at fungal contact sites on the time scale of minutes - a time

87 scale that is relevant to the earliest signaling events necessary for innate immune fungal

88 recognition. In the intensely studied immunologic synapse, it is known that

89 immunoreceptors in the T cell/Antigen Presenting Cell (APC) immune synapse are actively

90 transported into the synapse within minutes via their coupling to a centripetal

91 RHOA/Myosin II dependent actomyosin flow (AMF)[10]. Likewise, we previously

92 demonstrated that that Dectin-1 stimulation by glucan activates mechanical contractility 
93 signaling via a RHOA/ROCK/Myosin II signaling module within minutes post-stimulation

94 [11]. Thus, the central hypothesis tested in this study is that Dectin-1 activates a transport

95 mechanism, through RHOA/ROCK/Myosin II dependent signaling processes, which

96 facilitates the recruitment of DC-SIGN to the contact site. This would be expected to

97 improve fungal particle retention by providing higher avidity adhesive interactions with

98 the fungal cell wall.

99 We used a micropipette-micromanipulation approach to provide very high

100 spatiotemporal control over host-pathogen contact site formation. We report that Dectin-

101 1, in collaboration with DC-SIGN, does promote improved capture of C. albicans yeast.

102 This occurs through improved recruitment of DC-SIGN to the contact site in a manner that

103 is dependent upon Dectin-1 signaling via RHOA, ROCK and myosin II. These findings

104 provide a high-resolution view of early events in receptor recruitment processes that tailor

105 the earliest stages of the innate immune anti-fungal response.

106

107 2. Materials and Methods

$108 \quad 2.1$ Cell culture

109 HEK-293 cells (ATCC, \#CRL-1573) were cultured in DMEM containing 10\%

110 FBS, 1\% penicillin-streptomycin, $2 \mathrm{mM}$ L-glutamine and $1 \mathrm{mM}$ sodium pyruvate at $37^{\circ} \mathrm{C}$,

111 in a 5\% CO2 environment in an incubator. The identity and mycoplasma-free status of the

112 cell line was independently confirmed by submission to ATCC Human Cell Line 113 authentication (STR) and mycoplasma detection (PCR) services. 
mApple-Dectin1A-C-10 was a gift from Michael Davidson (Addgene plasmid \#

117 54883; http://n2t.net/addgene:54883; RRID:Addgene_54883). pEGFP-DC-SIGN was a

118 generous gift from Ken Jacobson[12]. pUNO1-hDectin-1a was purchased from Invivogen

119 (\#puno1-hdectin1a). mCardinal-Lifeact-7 was a gift from Michael Davidson (Addgene

120 plasmid \#54663). Transfection with plasmid was performed following standard protocols

121 for Fu gene 6 (Promega, \#E2691). Cells were selected for stable expression using Geneticin

122 (G418 sulfate) (Thermo Fisher Scientific, 10131035; for pEGFP-DC-SIGN) at $400 \mu \mathrm{g} / \mathrm{ml}$

123 or Blasticidin (Invivogen \#ant-bl-05; for pUNO1-hDectin-1a) at $20 \mu \mathrm{g} / \mathrm{ml}$ for 2 weeks.

124

$125 \quad 2.3$ Micropipette

$126 \quad 1.5 \mathrm{~mm}$ outer diameter and $1.12 \mathrm{~mm}$ inner diameter borosilicate glass capillaries

127 were purchased from World Precision Instruments (WPI\# TW150-4). We optimized

128 fabrication procedures to obtain micropipettes with $2 \mu \mathrm{m}$ diameter tips. We used a 129 microelectrode puller (WPI \#micrPUL-1000) for pulling micropipettes. Our final protocol

130 for pulling micropipette of $2 \mu \mathrm{m}$ was as follows.

\begin{tabular}{|l|l|l|l|l|}
\hline & Heat & Force & Distance & Delay \\
\hline 1 & 680 & 200 & 2.50 & 55 \\
\hline 2 & 600 & 170 & 1.50 & 60 \\
\hline 3 & 550 & 150 & 0.60 & 35 \\
\hline 4 & 500 & 100 & 0.30 & 20 \\
\hline
\end{tabular}

131 Table 1. Values of each parameter in WPI microelectrode puller used to pull $2 \mu \mathrm{m}$ diameter

132 tip micropipettes. 
135 C. albicans clinical isolate TRL035 was obtained as previously described[13].

136 Isolate was stored as single-use glycerol stock aliquots $-80^{\circ} \mathrm{C}$. This stock was transferred

137 to $5 \mathrm{ml}$ sterile yeast extract-peptone-dextrose (YPD) medium (Becton Dickinson) at

138 concentration of $1 \times 10^{5}$ cell/ml of YPD and grown for 16 hours at $30^{\circ} \mathrm{C}$, with a shaking

139 speed of $300 \mathrm{rpm}$. The glycerol stock contained $4 \times 10^{7}$ yeast $/ \mathrm{ml}$ and was previously

140 calibrated to provide $3 \times 10^{8} / \mathrm{ml}$ yeast cells at the late log phase under the stated growth

141 conditions.

142

\subsection{Silicone chambers}

144 We used silicone isolators (Grace Bio-labs \# 665116 and \# 665203) on cover glass

145 in configurations as shown below (Fig. 1). Whole chamber was sterilized by passing it

146 through a Bunsen burner blue flame 5 times.
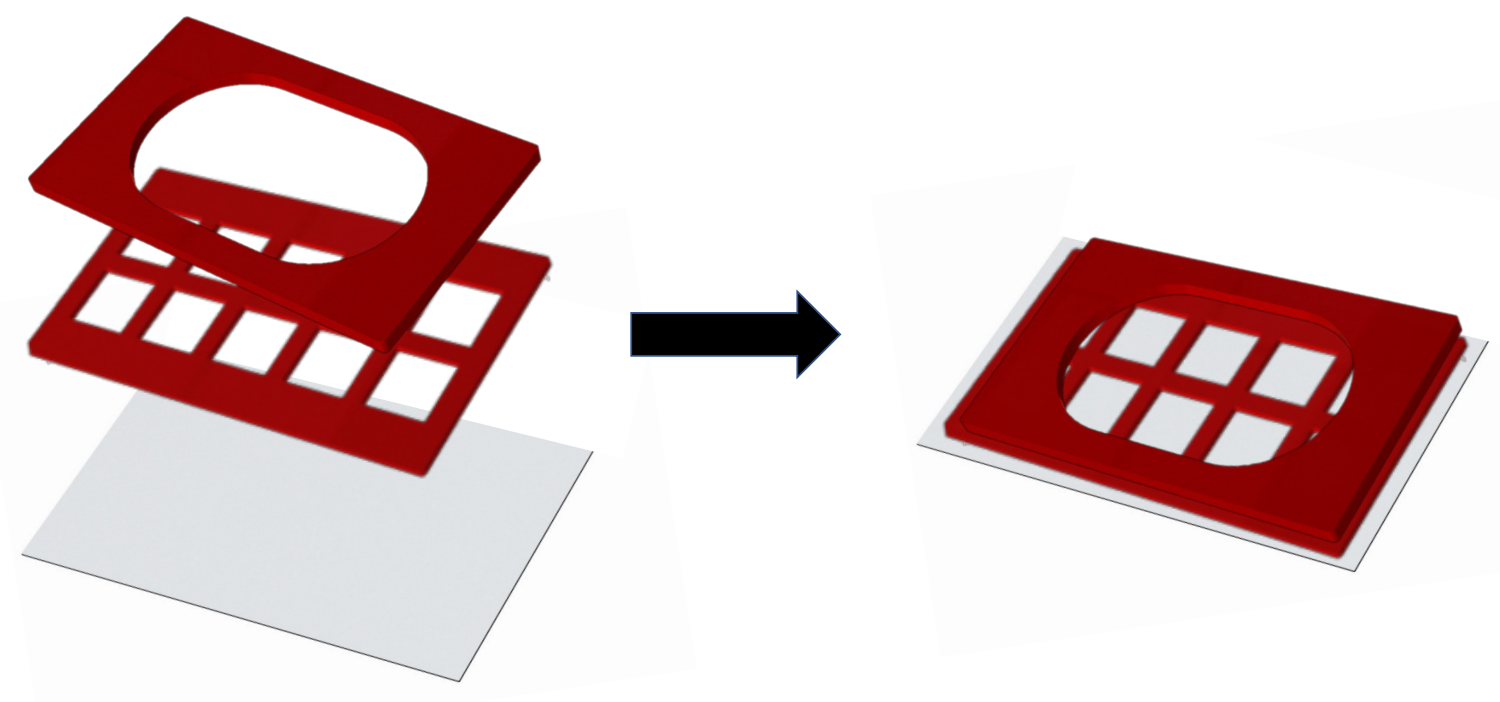
148 Figure 1. Arrangement of silicone chambers for growing HEK-293 cells for contact site 149 studies.

\subsection{Contact site studies}

153 generated, as described above. 2 days before each experiment mApple-Dectin1A-C-10

154 stable line cells were transiently transfected with pEGFP-DC-SIGN. Next day cells and

155 yeast were seeded into separate compartments of sterilized silicone chambers in a

156 configuration as shown below (Fig. 2).

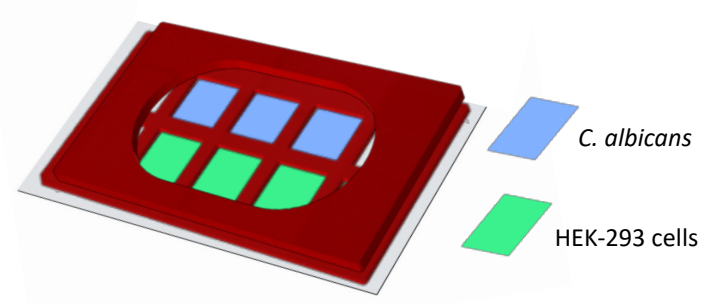

161 Figure 2. Separation of HEK-293 cells and yeast within chamber.

164 (Invitrogen, \#C10046). Original stock CMDR was freshly diluted 1:100 in culture medium

165 to form a working stock solution. $10 \mu$ working stock CMDR was added per $1 \mathrm{ml}$ of culture

166 medium in the culture vessel. Depending on the condition, along with CMDR, various

167 inhibitors were added during this stage. Blebbistatin (Sigma-Aldrich, \#203390) at $12.5 \mu \mathrm{M}$,

168 or Y-27632 (Sigma-Aldrich, \#Y0503) at $5 \mu \mathrm{M}$ were added for 1 hour. For RHO inhibitor 
169 conditions, cells were treated with C3 transferase (Cytoskeleton Inc., \#CT04) $1.5 \mu \mathrm{g} / \mathrm{ml}$ for

1702 hours. Both CMDR and inhibitor were added to culture dish at the same time. TRL035

171 C. albicans were stained with Fluorescent Brightener 28 (Calcofluor White) (Sigma-

172 Aldrich \#F3543). $25 \mu \mathrm{l}$ of $1 \mathrm{mg} / \mathrm{ml}$ Calcofluor White was used to stain $1 \mathrm{ml}$ of yeast in

173 PBS pH 7.4 for 15 min. Then yeast were washed three times with PBS and vortexed for 15

174 minutes. C. albicans were added to smaller chambers as shown in Figure 2. Glucose

175 oxidase (Sigma-Aldrich \# G2133) and catalase (Sigma-Aldrich \# C100) were added to the

176 chamber at concentration of $0.5 \mathrm{mg} / \mathrm{ml}$ and $40 \mu \mathrm{g} / \mathrm{ml}$, respectively, during data acquisition

177 to reduce photobleaching.

178 Micropipettes were filled with PBS using MicroFil (WPI \#MF28G-5) and

179 syringe. The PBS-filled micropipette was then attached microelectrode holder (WPI \#

180 MPH415). The inlet of the microelectrode holder was attached to $1 \mathrm{ml}$ syringe using Luer

181 lock via plastic tubing.

182 For micromanipulation, we used a Sensapex micromanipulator. We attached

183 microelectrode holder to micromanipulator using an electrode handle (WPI \#2505). The

184 silicon chamber was then placed on the FV1000 laser-scanning confocal microscope

185 (Olympus, Center Valley, PA) with controlled temperature, $37^{\circ} \mathrm{C}$ at $5 \% \mathrm{CO}_{2}$. We used a

186 60x super-corrected, 1.40 NA, Plan-Apochromat oil immersion objective for imaging cells.

187 We replaced one microscope eye piece with a Centering Telescope eyepiece (Olympus \#

188 U-CT30-2), which allowed a separate focal plane in each eyepiece for ease in positioning 189 the micropipette.

190 We identified a suitable single C. albicans yeast for capture and then adjusted the 191 microscope's focal plane to $20 \mu \mathrm{m}$ above that yeast. We focused the Centering Telescope 
192 eyepiece on the tip of micropipette. The telescope eyepiece was then focused at a plane

193 closer to the yeast, then we lowered the tip of micropipette via micromanipulator into the

194 lower focal plane toward the yeast, while being monitored through the telescopic eyepiece.

195 This process was repeated till we reached the level of fungus. This was done to avoid

196 breaking of micropipette tip while lowering it. We always lowered telescopic eyepiece

197 focus first and then adjusted micropipette level. Once at the same level as C. albicans, we

198 applied negative pressure using the syringe to capture a single yeast on the micropipette

199 tip. Then, we manipulated C. albicans to the chamber with HEK-293 cells by moving from

200 the yeast chamber, over the isolator barrier, and translating into the cell chamber, keeping

201 the tip submerged at all times. Finally, C. albicans was brought near the plasma membrane

202 of a HEK-293 cell expressing the appropriate receptors, as verified by their fluorescent

203 protein tags.

204 Confocal fluorescence microscopic observation of contacts sites was conducted

205 with the following parameters. Calcofluor White (a marker for all yeast) was excited with

206 a $50 \mathrm{~mW}, 405 \mathrm{~nm}$ diode laser operated at 1\% power, and CMDR was excited with a 20

$207 \mathrm{~mW}, 635 \mathrm{~nm}$ diode laser operated at 0.5\% power. EGFP-DC-SIGN was excited with a 20

$208 \mathrm{~mW}, 473 \mathrm{~nm}$ diode laser operated at 1\% power. mApple-Dectin-1 was excited with a 20

$209 \mathrm{~mW} 559 \mathrm{~nm}$ laser at $1 \%$ power. These lines were reflected to the specimen by a

$210405 / 473 / 559 / 635$ multi-edge main dichroic element and routed through a confocal pinhole

211 (110 mm diameter) to secondary dichroic followed by bandpass emission filters in front of

212 two independent PMT detectors and two independent high-sensitivity GaAsP PMT

213 detectors (HSD). Specifically, the emission light passed by the main dichroic was directed

214 to PMT1 (Calcofluor White channel) via reflection from the SDM473 dichroic and passage 
215 through a BA430-455 nm bandpass filter. For the CMDR channel, light from SDM473 was

216 directed to a $640 \mathrm{~nm}$ shortpass dichroic and BA575-675 $\mathrm{nm}$ bandpass filter. Light from

$217640 \mathrm{~nm}$ shortpass dichroic was directed to SDM560 filter cube to HSD1 (the EGFP-DC-

218 SIGN channel) via passage through a BA490-540 nm bandpass filter. For mApple-Dectin-

219 1, light was directed via SDM560 filter cube to HSD2 via passage through a BA575-675

$220 \mathrm{~nm}$. 60x lens with 3x zoom was used to capture images. Further a subregion of interest for

221 image scanning was selected such that the region was small enough to be scanned at a rate

222 of $0.400 \mathrm{sec}$ per frame. Overall, pixel size for all images was 7.24 pixels per micron.

223 Imaging was started and then, with the micromanipulator, contact was made between $C$.

224 albicans and HEK-293 cells. The contact site was imaged for 10 minutes total duration

225 after contact initiation.

\subsection{Polystyrene bead control for contact site studies}

228

For making Dextran coated beads, we used $5 \mu \mathrm{m}$ streptavidin-coated polystyrene

229 bead (Spherotech \#VP-60-5). We used 1,1'-carbonyldiimidazole in DMSO based system

230 as described by Tam et al. to conjugate dextran (Sigma-Aldrich \# 31388) with beads. Rest

231 of the procedure for making contact and imaging was exactly same for TRL035 $C$.

232 albicans.

233

2342.8 FRAP studies

235 For FRAP studies, exact same steps as mentioned above for contact site studies

236 were followed. Then TRL035 contact site was allowed to mature for $10 \mathrm{~min}$. after contact.

237 Then a rectangular FRAP window was selected so that it included the whole of the contact 
238 site. Imaging was started and 5 frames were collected pre-bleach. Then, we photobleached

239 the contact area with $473 \mathrm{~nm}$ and 559 laser, 100\% power for 500 milliseconds. Imaging

240 was continued for $10 \mathrm{~min}$. to quantify recovery. FRAP analysis was done using

241 easyFRAP[14].

242

2432.9 Analysis of contacts site data

244 For quantifying the contact site, we used the Fiji distribution of ImageJ. We

245 demarcated overlapping pixels between dilated calcofluor channel and CMDR channel.

246 These overlapping pixels denote the contact site. All further calculation of MFI (Mean

247 Fluorescence Intensity) for DC-SIGN, Dectin-1 channel and their normalizations were

248 done from these contact site pixels only. The detailed steps followed were as follows.

249 Calcofluor white (405 channel) was thresholded to make a fungal mask and converted to

250 binary. The binary fungal mask was then dilated 2 times. The fungal mask was divided by

251255 to make all pixel values 1 . This is essential for calculating overlapping areas in next

252 steps. The CMDR (635 channel) was thresholded and converted to binary to create a

253 CMDR mask. The CMDR mask was divided by 255. The fungal mask was multiplied by

254 CMDR mask to mark overlapping pixels as those demarcating the contact site mask, within

255 which each pixel had a numerical value of 1 . The remaining non-mask pixels had a

256 numerical value of 0 . Overlapping pixels were multiplied with Dectin-1, DC-SIGN and

257 CMDR raw pixel intensities, creating masked Dectin-1, DC-SIGN and CMDR datasets.

258 RawDensity, which is the sum of intensities of all pixels in a dataset, was calculated for

259 each the contact site masked DC-SIGN, Dectin-1 and CMDR datasets. The same

260 calculation was performed for the contact site mask image, which provides the area (pixels) 
261 of the contact site mask. Mean Fluorescence Intensity (MFI) per pixel for DC-SIGN, and

262 Dectin-1, and CMDR were calculated by dividing RawDensity for each of these datasets

263 by the contact site area in pixels. To normalize for variable amount of membrane in a

264 contact site, we divided MFI of DC-SIGN and Dectin-1 by the corresponding contact site

265 CMDR MFI. Finally, to control for possible differential expression/staining of individual

266 HEK-293 cells, we expressed the above normalized receptor MFI signals as a percentage

267 of their value at time 0 , on a per cell basis.

268

$$
M F I=\left[\frac{\frac{\text { Receptor MFI at time } x}{\text { CMDR MFI at time } x}}{\frac{\text { Receptor MFI at time } 0}{\text { CMDR MFI at time } 0}}\right] * 100
$$

For the yeast capture assay, we used HEK-293 cells stably transfected mApple-

273 Dectin1A-C-10 cells were used or transiently transfected with pEGFP-DC-SIGN. For DC-

274 SIGN only condition parental cells were transiently transfected with pEGFP-DC-SIGN.

275 Overall, the following 4 conditions were used for experiments, 1) EGFP-DC-SIGN +

276 mApple-Dectin-1, 2) EGFP-DC-SIGN, 3) mApple-Dectin-1, 4) Untransfected conditions.

277 Cells were seeded at $2.5 \times 10^{4} /$ dish, overnight in $35 \mathrm{~mm}$ Mattek dishes. Then TRL035 $C$.

278 albicans stained with calcofluor white were added to dishes at $10 \times 10^{4} / \mathrm{dish}$. Then dishes

279 were kept on rocker shaker (BR200 2d rocker, Southwest Science) for $30 \mathrm{~min}$. Cells were

280 then washed 3 times with PBS and fixed with 4\% PFA, and the number of fungi attached

281 to each cell of interest were counted under microscope with imaging condition similar to

282 described in contact site studies. Binding studies with cells expressing both DC-SIGN and

283 Dectin-1 enumerated yeast binding only to cells confirmed to co-express both receptors. 


\subsection{TIRF microscopy}

HEK-293 cells were stably transfected with pUNO1-hDectin-1a and mCardinal-

287 LifeAct-7. 1 days before experiment these cells were transiently transfected with EGFP-

288 DC-SIGN. Cells were dissociated from dish surfaces using brief exposure to $0.25 \%$

289 trypsin-EDTA followed by addition of protein rich medium to rapidly quench trypsin

290 activity and washing into fresh culture medium. Then these cells were put on $35 \mathrm{~mm}$ dish

291 coated with $\beta$-glucan. Cells were allowed to settle down on these surfaces in incubator for

29230 min. Then cell membrane was observed under Olympus IX83 TIRF/Single Molecule

293 Localization Microscope. $488 \mathrm{~nm}$ and $561 \mathrm{~nm}$ lasers were used to excite EGFP-DC-SIGN

294 and mCardinal-LifeAct respectively. Cell membrane was observed for $5 \mathrm{~min}$. To address

295 the potential concern that trypsinization would damage transmembrane receptors

296 sufficiently to render cells non-responsive to fungal ligands, we loaded cells with Fluo-4

297 calcium dye, trypsinized and settled them on glucan coated glass as above, and observed

298 calcium flux upon Dectin-1 contact with glucan coated glass. We observed calcium flux

299 on glucan coated glass but not on glucan free glass surfaces, demonstrating that the cells

300 remained functional following trypsinization (data not shown)

301

302 2.12 Coating dishes with $\beta$-glucan

303 The central glass region of Mattek glass bottom $35 \mathrm{~mm}$ dishes was coated with $\beta$-glucan

304 to permit TIRF microscopic observations of early contact site membrane dynamics using

305 a coupling chemistry previously reported by Tam, et al.[6]. The following procedure was

306 used to produce these surfaces. $200 \mu 1$ of $0.01 \mathrm{w} / \mathrm{v}$ Poly-1-lysine aqueous solution (Sigma, 
307 P4707) was put on the central portion of $35 \mathrm{~mm}$ dishes and adsorption was permitted to

308 occur for $30 \mathrm{~min}$. Excess solution was removed and dishes were washed with DMSO for 3

309 times. Poly-l-lysine amines were activated by immersion in $0.5 \mathrm{M} 1,1^{\prime}-$

310 Carbonyldiimidazole (CDI; Sigma, 115533) in DMSO for 1 hour. Dishes were washed

311 with DMSO 3 times. $10 \mathrm{mM}$ Medium Molecular Weight (145 kDa) $\beta$-(1,3)-glucan from

312 ImmunoResearch Inc. (Eagan, MN) in DMSO was added to the dishes. The reaction was

313 allowed to incubate overnight, excess solution was removed, and dishes were washed with

314 water 3 times.

315

3162.13 Phagocytosis assay

317 TRL035 C. albicans were first stained with Fluorescent Brightener 28 (calcofluor white)

318 (Sigma-Aldrich \#F3543). $25 \mu \mathrm{lof} 1 \mathrm{mg} / \mathrm{ml}$ calcofluor white was used to stain $1 \mathrm{ml}$ of 16

319 hour fungal culture in PBS (Gibco) pH 7.4 for 15 minutes. Then cells were washed 3 times

320 with PBS and stained with $75 \mu \mathrm{M}$ CypHer5E NHS ester (GE Healthcare, PA \#15401) for

3211 hour at $25^{\circ} \mathrm{C}[13]$. C. albicans were then added to culture dishes with HEK-293 cells

322 transiently transfected with pEGFP-DC-SIGN for 1 hour and observed under a microscope

323 for increased fluorescence in CypHer5E channel as an indicator of fungi which had been

324 phagocytosed.

325

$326 \quad 2.14$ Agent Based Modeling

327 An agent-based model of DC-SIGN transport into contact sites was created in the Netlogo

328 modeling environment. Source code and a full description of the parameterization of this

329 model are provided in Supplemental Methods. 
3313.1 Dynamics of Dectin-1 and DC-SIGN recruitment to a contact site for capture of C.

332 albicans

333 We examined the possible role of Dectin-1 and/or DC-SIGN in the capture of

334 fungal particles using a C. albicans yeast capture assay. This assay was designed to test the

335 ability of cells expressing these receptors, alone or in combination, to bind and stably

336 capture yeast after a relatively brief exposure to particles under conditions of fluid shear.

337 To unambiguously measure fungal capture attributable to these receptors, we used a

338 HEK293 cell system with no endogenous expression of relevant PRRs, which was then

339 transfected to express one or both receptors. We found that, within $30 \mathrm{~min}$., cells captured

340 an average of 5.1, 1.62, 2.23 and 0.23 C. albicans fungal particles per cell under DC-SIGN

341 + Dectin-1, Dectn-1 only, DC-SIGN only and untransfected/parental HEK-293 conditions,

342 respectively (Figure 3a). We found significantly increased capture of fungal particles in the

343 co-expressed condition compared to the condition with either individual receptor expressed

344 alone. This effect was slightly more than additive. Given the normal distribution of yeast

345 capture measured in co-expressing cells, we found that there is only $3.2 \%$ probability to

346 find a fungal capture equivalent to the sum of means of yeast capture measured for cells

347 expressing DC-SIGN and Dectin-1 alone. We hypothesized that DC-SIGN and Dectin-1

348 collaborate to improve capture of C. albicans yeast in rapidly developing contact sites with

349 this pathogen.

350 To look for receptor recruitment dynamics at the contact site, we used micropipette-

351 micromanipulator based system where a single fungal particle was adhered to the tip of the

352 micropipette and advanced into contact with the cell to form a single host-pathogen contact 
353 site at a well-defined location and time (Figure 3b). Fluorescent protein tagged receptors'

354 recruitment to the contact site was followed by live cell confocal fluorescence microscopic

355 observation of the contact site from initiation to 10 minutes post-contact and reported as a

356 relative increase over receptor density at the time of contact site initiation. We additionally

357 reported changes in cumulative receptor intensity (sum of all pixel intensities in contact)

358 and contact site area. First, we measured recruitment of EGFP-DC-SIGN or mApple-

359 Dectin-1A individually expressed in HEK293 cells to contact sites formed as described

360 above. In the DC-SIGN only condition, we found a $35.04 \%$ increase in normalized density,

$36170.76 \%$ increase in cumulative receptor intensity and $49.83 \%$ increase in the contact site

362 area (Figure 3c). For Dectin-1 only expression, we found no significant increase in

363 normalized Dectin-1 density, cumulative receptor fluorescence and contact site area

364 (Figure 3d). Then, we examined contact sites in HEK-293 cells co-transfected with both

365 EGFP-DC-SIGN and mApple-Dectin-1A (Figure 3e). In dual expressing cells, we found a

366 significant increase in normalized DC-SIGN density of $103.15 \%$ compared to pre-contact

367 DC-SIGN density. We did not find a significant increase in Dectin-1 density within 10

368 minutes post contact in dual expressing cells, similarly to the observation in Dectin-1 only

369 expressing cells. For total amount of receptor in contact sites, we found $154.15 \%$ and

$37045.33 \%$ increase in cumulative receptor intensity for DC-SIGN and Dectin-1 respectively.

371 We also found $57.97 \%$ increase in contact area in dual expressing cells. With control

372 dextran coated polystyrene beads, we did not find any increase in DC-SIGN or Dectin-1

373 recruitment (Figure 3f). In summary, we found that DC-SIGN exhibited significantly

374 increased recruitment and accumulation at contact site in the presence of Dectin-1 within 
bioRxiv preprint doi: https://doi.org/10.1101/2020.12.15.422964; this version posted December 15, 2020. The copyright holder for this preprint (which was not certified by peer review) is the author/funder, who has granted bioRxiv a license to display the preprint in perpetuity. It is made available under aCC-BY-NC-ND 4.0 International license.

375 the $1^{\text {st }} 10$ min of C. albicans contact. Whereas, Dectin-1 did not show recruitment within

376 the $1^{\text {st }} 10$ min of $C$. albicans contact.

a

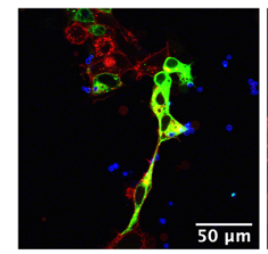

DC-SIGN + Dectin-1

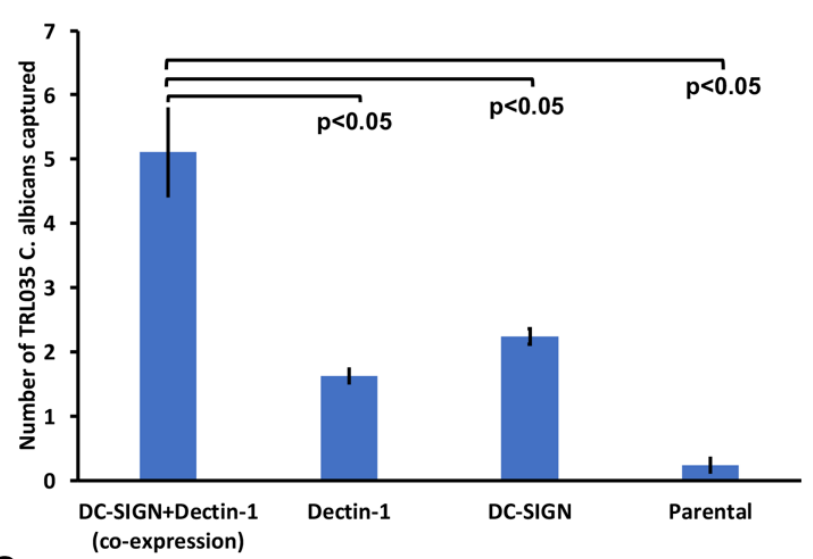

C

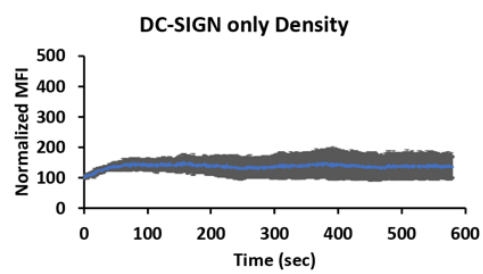

d

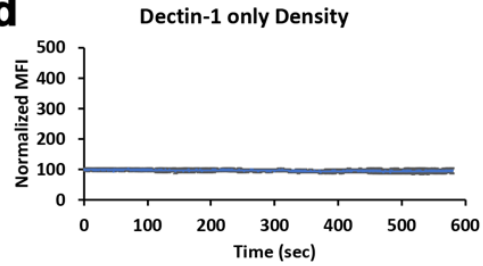

DC-SIGN only Total DC-SIGN

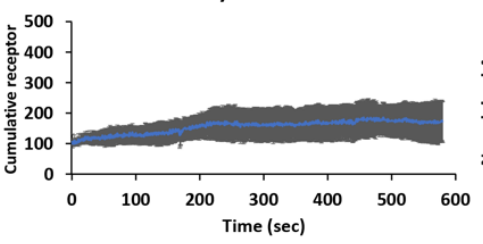

Dectin-1 only Total Dectin-1

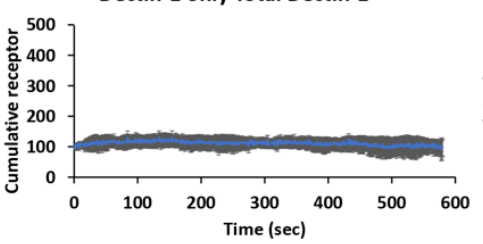

Parental

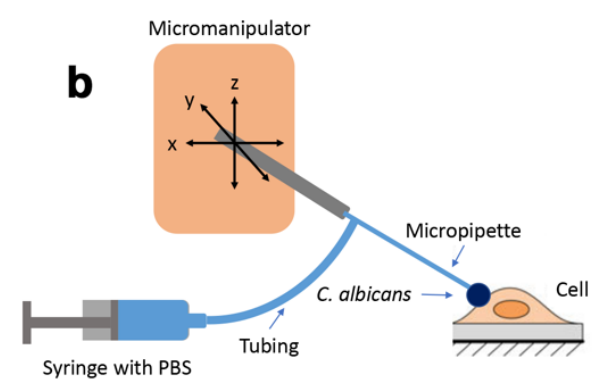

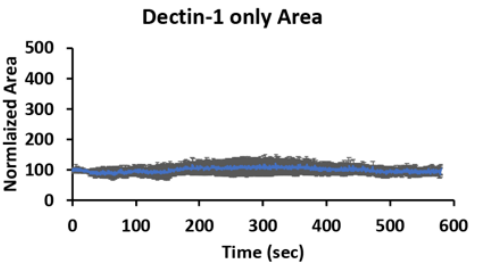



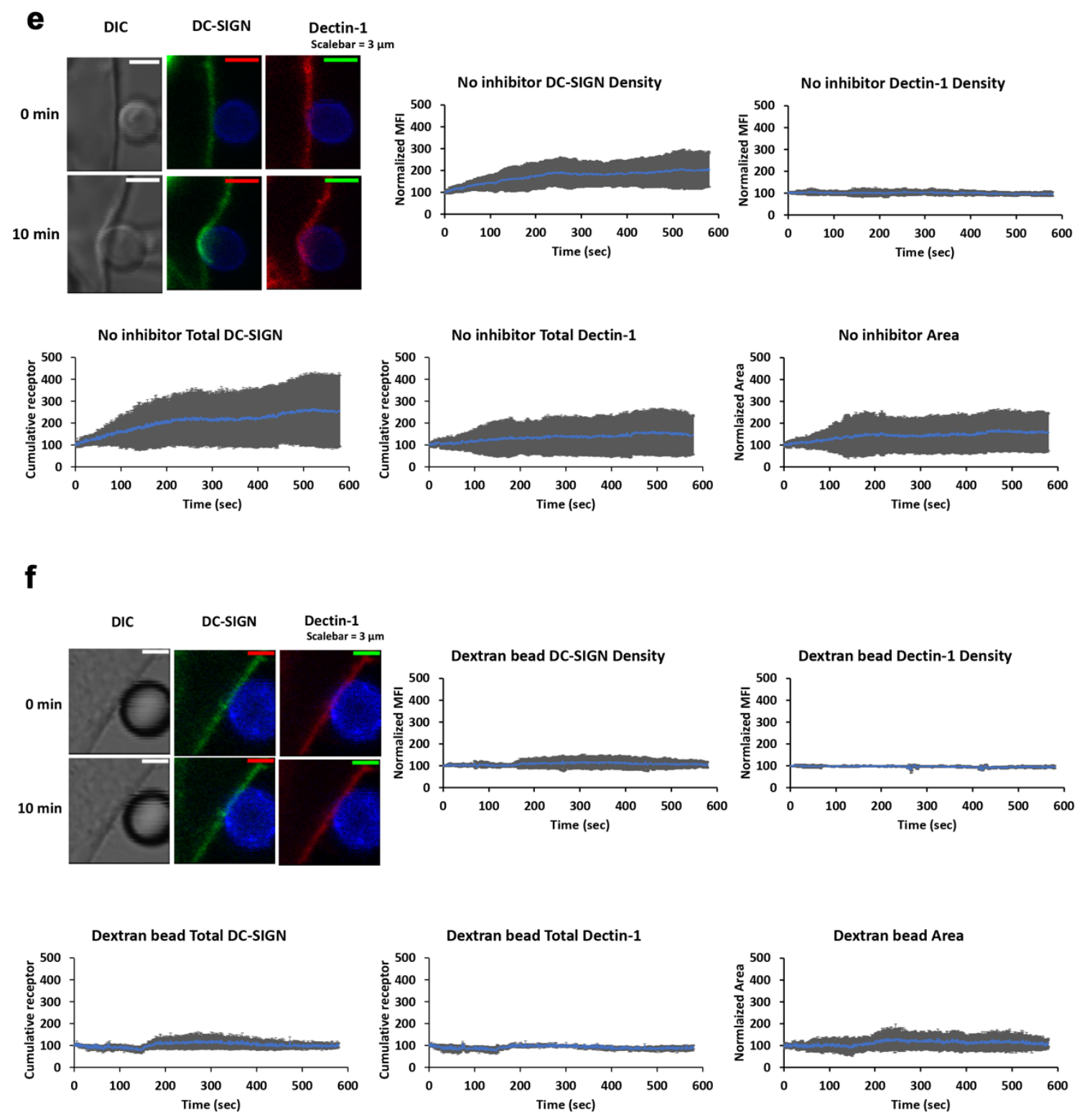

379 Figure 3. Dynamics of DC-SIGN and Dectin-1 recruitment to a contact site for capture of

380 C. albicans. (a) TRL035 C. albicans (blue) capture assay by HEK-293 cells transfected

381 with EGFP-DC-SIGN (green) and/ mApple-Dectin-1 (red) (n=61), Dectin-1 only ( $\mathrm{n}=63)$,

382 DC-SIGN only $(\mathrm{n}=43)$ and parental $(\mathrm{n}=49)$. Average number of fungal particles captured

383 by a cell under each condition is denoted in the graph (the reported value of " $\mathrm{n}$ " denotes

384 the number of total cells pooled from $\geq 3$ independent experimental replicates). (b) 
385 Schematic diagram of micropipette-micromanipulator system. (c \& d) Dynamics of DC-

386 SIGN (panel c, $n=6$ ) or Dectin-1 (panel d, $n=6$ ) density enrichment at the contact site under

387 individual receptor expression conditions. (e) Images showing contact of TRL035 $C$.

388 albicans (blue) with HEK-293 cells transfected with EGFP-DC-SIGN (green) and

389 mApple-Dectin-1 (red) at time 0 and time $10 \mathrm{~min}$. Graphs show dynamics of normalized

390 DC-SIGN and Dectin-1 density enrichment, total cumulative receptor intensity and contact

391 site area under co-expression condition of receptors at TRL035 contact site $(n=10)$. (f)

392 Dynamics of EGFP-DC-SIGN (green) and mApple-Dectin-1 (red) density enrichment

393 under co-expression of receptors at contact site with dextran coated polystyrene bead (blue)

$394(n=3)$. All graphs represent the mean \pm S.D. for the indicated value at each time point).

3.2 Actomyosin based active recruitment of DC-SIGN at C. albicans contact site.

397 We previously reported a novel signaling connection between Dectin-1 activation

398 and generation of cellular mechanical forces via stimulation of actomyosin contractility

399 [11]. Actomyosin contractility has previously been found to generate actomyosin flows

400 (AMFs) that support active transport of plasma membrane proteins (i.e., TCR and BCR

401 transport at immunological synapses, [10]). Therefore, we next looked for possible

402 involvement of the Dectin-1 activated actomyosin contractility signaling mechanism in

403 early active recruitment of DC-SIGN to fungal contact sites. We hypothesized that Dectin-

4041 mediated actomyosin contraction leads to an AMF at host-pathogen contact sites, and

405 that DC-SIGN coupling to this AMF is important for recruitment of DC-SIGN to $C$.

406 albicans contact sites. This is feasible because Dectin-1 signaling gives rise RHOA-

407 ROCK-Myosin II activation, leading to actomyosin contractility[11]. So, inhibition of this 
408 signaling mechanism would be expected to abrogate the development of a hypothesized

409 AMF and any active receptor recruitment connected to it. We pretreated HEK-293 cells

410 transfected with DC-SIGN and Dectin-1 with various inhibitors of the actomyosin

411 contractility signaling axis. We found that cells treated with inhibitors of RHOA, ROCK

412 and Myosin-II exhibited normalized DC-SIGN density of 41.47\%, 62.96\% and 44.04\%

413 respectively at 10 minutes post-contact formation (Figure 4a). Thus, there was significantly

414 less recruitment of DC-SIGN in the presence of each of the 3 inhibitors relative to the

415 untreated condition. For total cumulative DC-SIGN intensity within the contact sites, we

416 found an increase of $71.13 \%, 78.79 \%$ and $43.03 \%$ with RHOA, ROCK and Myosin-II

417 inhibitor (Figure 4b). Thus, showing less total accumulation of DC-SIGN in inhibitor

418 treated contacts as well. Concomitantly, we observed contact site areas increased by

$419121.77 \%, 105.43 \%$ and 18.36\% with RHOA, ROCK and Myosin-II inhibitor, respectively,

420 at 10 minutes post-contact formation (Figure 4c). Thus, there was an increase in contact

421 area with RHOA and ROCK inhibitor, but a decrease in contact area with Myosin-II

422 inhibitor compared to no inhibitor condition.

423 

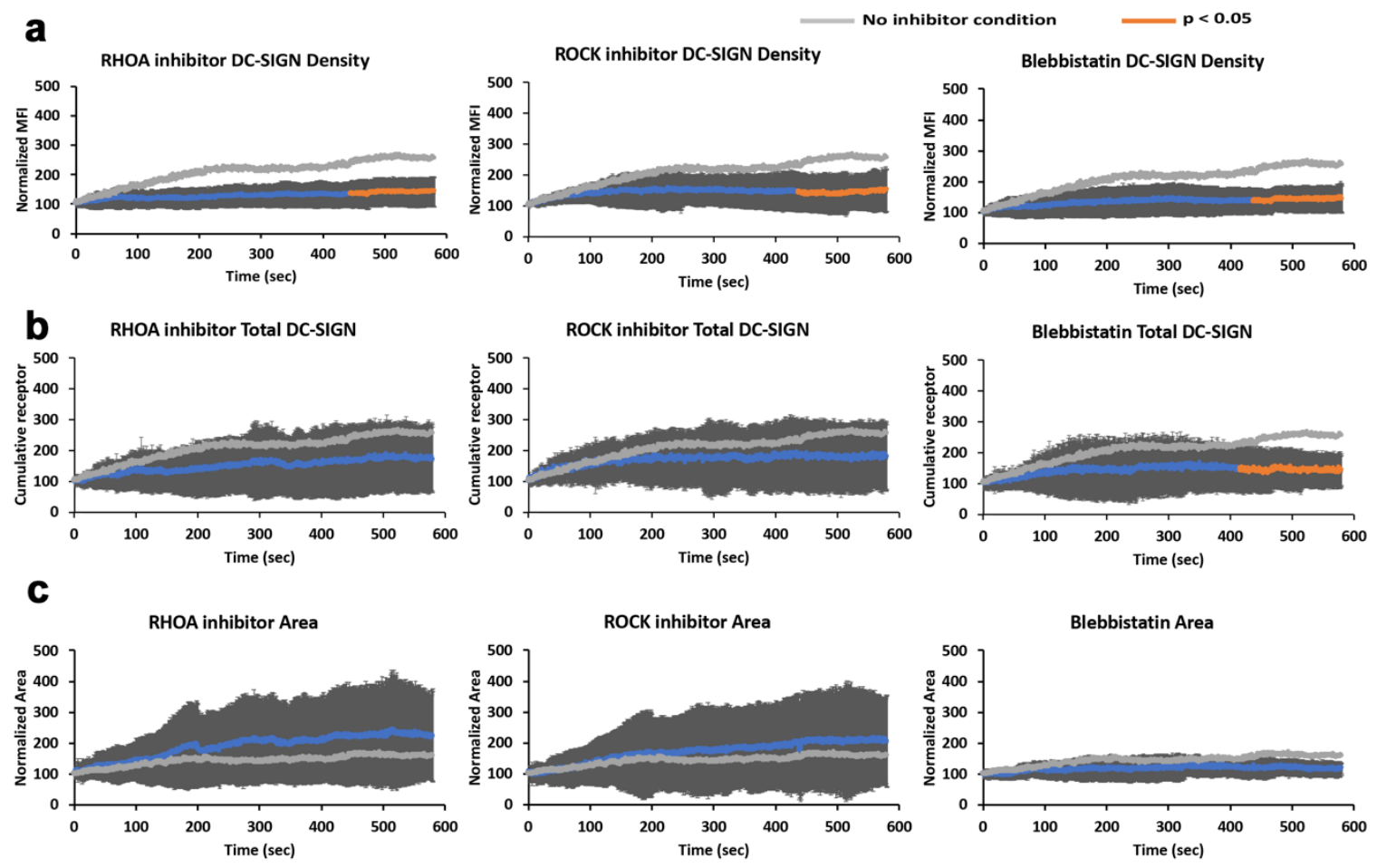

425 Figure 4. Role of RHOA-ROCK-Myosin II based system in recruitment of DC-SIGN

426 under co-expression condition of Dectin-1 and DC-SIGN at TRL035 C. albicans contact

427 site. (a) Normalized receptor density enrichment in fungal pathogen contact sites for DC-

428 SIGN at the end of 10 min. under RHOA $(n=11)$, ROCK $(n=9)$ and Myosin-II $(n=9)$

429 inhibitory conditions, with comparison to the same in untreated cells. (b) Total cumulative

430 DC-SIGN intensity enrichment under the same conditions as panel a. (c) Contact site area

431 changes under the same conditions as panel a. All graphs represent the mean \pm S.D. for

432 the indicated value at each time point. 
438 arrived at the contact site. We hypothesized that receptor engagement with fungal cell wall

439 ligands after transport into the contact site would result in reductions in apparent receptor

440 lateral mobility, especially for DC-SIGN due to its multivalent binding to cell wall

441 mannoproteins. We used FRAP (Fluorescence Recovery After Photobleaching) on contact

442 sites after 10 minutes of contact site maturation (Figure 5a). Within contact sites, we found

443 that DC-SIGN showed $28 \%$ mobile fraction with recovery $\mathrm{t}_{1 / 2}$ of $66.19 \mathrm{sec}$ and Dectin-1

444 showed much larger $79 \%$ mobile fraction with recovery $t_{1 / 2}$ of $42.13 \mathrm{sec}$ (Figure $5 \mathrm{~b}$ ). On

445 statistical comparison of this contact site FRAP data we found that, relative to Dectin-1

446 values, DC-SIGN had lower lateral mobility $(\mathrm{p}=0.01)$ and exhibited a smaller mobile

447 fraction $(\mathrm{p}=0.0003)$. For non-contact membrane, we found that DC-SIGN showed 78.82\%

448 mobile fraction with recovery $\mathrm{t}_{1 / 2}$ of $35.13 \mathrm{sec}$ and Dectin-1 showed $79.66 \%$ mobile

449 fraction with recovery $\mathrm{t}_{1 / 2}$ of $14.08 \mathrm{sec}$ (Figure $5 \mathrm{c}$ ). On statistical comparison of DC-SIGN

450 within contact sites with non-contact DC-SIGN, we found a significant increase in recovery

$451 \mathrm{t}_{1 / 2}(\mathrm{p}=0.005)$ and significant decrease in mobile fraction $(\mathrm{p}=0.0002)$. For Dectin-1, we

452 found significant decrease in recovery $\mathrm{t}_{1 / 2}$ compared to non-contact membrane $(\mathrm{p}=0.001)$

453 and no significant difference for mobile fraction $(p=0.39)$. These findings indicate that

454 DC-SIGN is far less mobile than Dectin-1 within contact sites. This conclusion is

455 consistent with the expectation that highly multivalent DC-SIGN/N-mannan interactions

456 are likely to result in largely irreversible adhesive interactions of DC-SIGN nanodomains

457 with the outer cell wall surface of C. albicans - the likely physical basis of this receptor's

458 importance for rapid and effective capture of yeast. In contrast, Dectin-1 is primarily

459 monovalent [15] as it enters the contact site, and sites of glucan exposure are quite small

460 and sparsely distributed [16]. Thus Dectin-1 remains fairly mobile within the contact site, 
461 which may increase the efficiency of its search for rare sites of glucan exposure even

462 though $t_{1 / 2}$ is decreased in the contact as expected from Dectin-1 interactions with available

463 glucans at contact sites.

464 To rule out effects of RHOA inhibitor itself on receptor lateral mobility, we

465 did FRAP studies on cells exposed to RHOA inhibitor (Figure S2). These cells did not have

466 fungal contact sites to facilitate comparison of effects on basal receptor mobility of the

467 receptors. On comparing DC-SIGN lateral mobility in RHOA inhibited cells to untreated

468 controls, we found no significant difference in DC-SIGN recovery $\mathrm{t}_{1 / 2}(\mathrm{p}=0.66)$ or mobile

469 fraction $(p=0.71)$. The same was true for Dectin- 1 , with no significant difference in $t_{1 / 2}$

$470(p=0.53)$ or mobile fraction $(p=0.22)$. We conclude that RHOA pathway inhibition does

471 not impact the lateral mobility of DC-SIGN or Dectin-1, making such a direct effect an

472 unlikely contributor to the recruitment of these receptors to the contact site. 


\section{a}
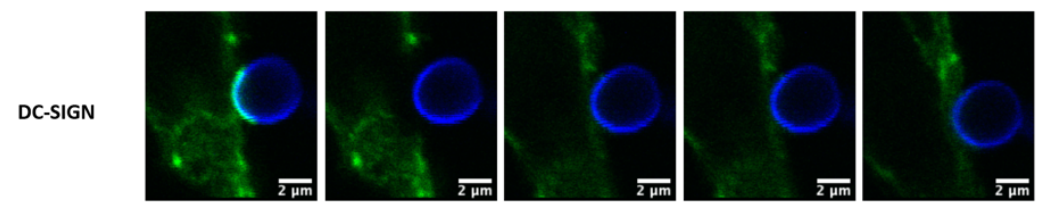

Pre-bleach frame

Bleach frame

$200 \mathrm{sec}$

$400 \mathrm{sec}$

$600 \mathrm{sec}$
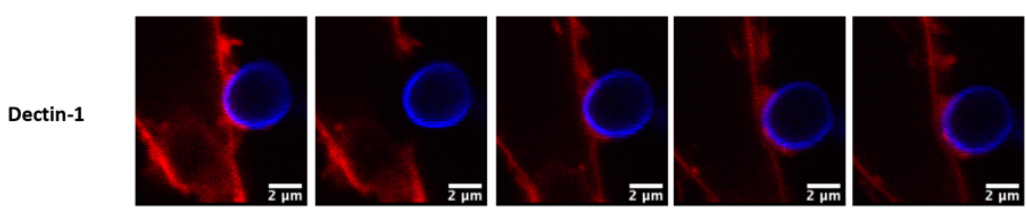

b
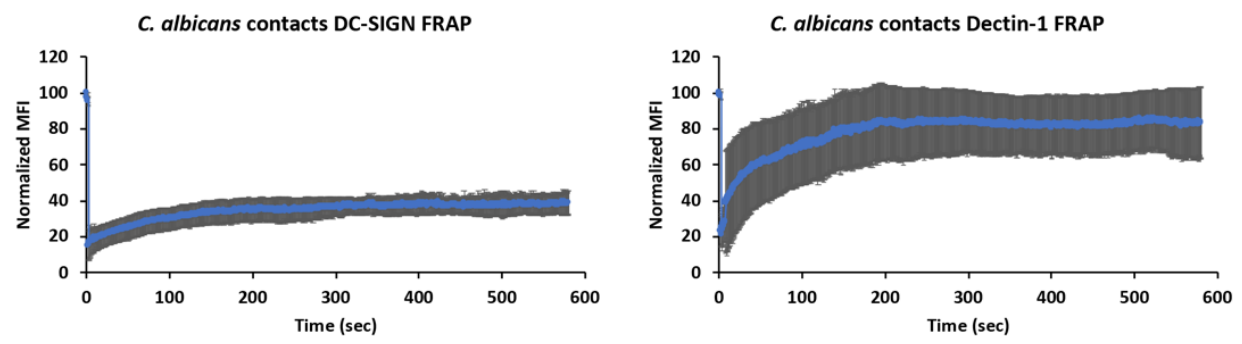

C
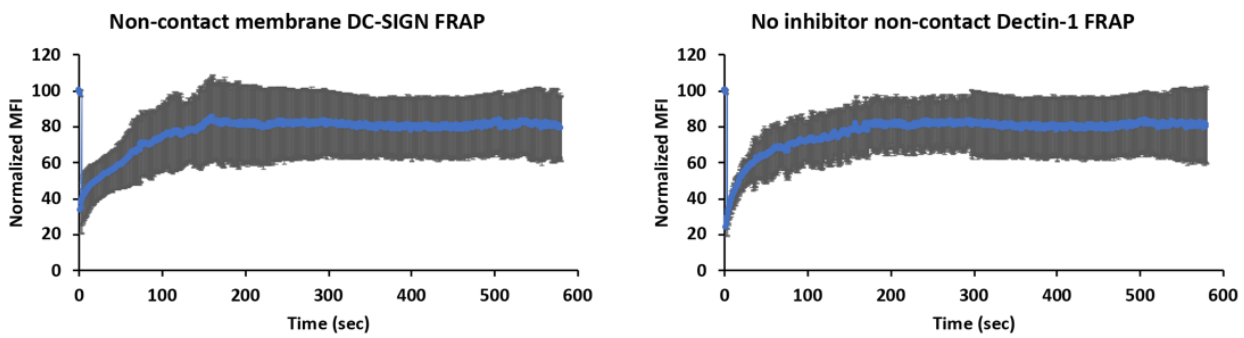

475

476 Figure 5. Lateral mobility characteristics of DC-SIGN and Dectin-1 at C. albicans inside

477 and outside of contact site zones. (a) Example pre/post-FRAP images of TRL035 $C$.

478 albicans (blue) contacts with HEK-293 cells transfected with EGFP-DC-SIGN (green) and

479 mApple-Dectin-1 (red). (b) Mean FRAP recovery curves of DC-SIGN (n=7) and Dectin-1

$480(\mathrm{n}=7)$ at contact sites and (c) non-contact membrane DC-SIGN $(n=6)$ and Dectin-1 $(n=6)$

481 with fluorescence recovery observed for $10 \mathrm{~min}$. All contact sites were allowed to mature

482 for $10 \mathrm{~min}$. after C. albicans contact before initiation of FRAP experiments. All graphs

483 represent the mean \pm S.D. for the indicated value at each time point. 
487 which have been described to undergo Brownian diffusion as well as apparent directed

488 transport in some contexts[17-19]. For instance, DC-SIGN nanodomain tracking studies

489 have reported that this receptor can undergo rapid, linear transport in the plane of the

490 plasma membrane, suggesting that some directed mobility of DC-SIGN is

491 possible[12,18,20,21]. As previously mentioned, directed transport at immunological

492 contact sites (e.g., T cell or B cell synapses) often involves cytoskeleton/motor protein

493 dependent centripetal motion of receptors toward the center of the contact site[10]. So, we

494 proposed that similar mechanism might exist at the fungal contact site. Therefore, we next

495 used an agent based model to test the hypothesis that a combination of Brownian diffusion

496 and periods of directed transport into the contact site could explain observed DC-SIGN

497 recruitment dynamics ("Directed + Brownian transport" model).

498

We created an agent-based computational model in the Netlogo modeling

499 environment, which was capable of predicting DC-SIGN accumulation rates in contact

500 sites wherein the DC-SIGN experienced a variable coupling to a centripetally directed

501 active transport mechanism (i.e., AMF), interspersed with regimes of Brownian diffusion

502 when uncoupled to the directed transport mechanism. This model was parameterized with

503 literature reported measurements and systematic parameter studies, as described in

504 Supplemental Methods. The key parameter in this model determining the degree of active 505 transport of DC-SIGN was the AMF coupling coefficient, which we systematically varied

506 to define a range of predicted DC-SIGN contact site recruitment. The Directed+Brownian

507 transport model was able to predict a DC-SIGN contact site recruitment rate of $108.72 \%$ 
508 increase over pre-contact density (Figure 6), similar to that observed experimentally in

509 cells expressing DC-SIGN and Dectin-1 with 103.15\% increase over pre-contact density

510 (Figure 3e). To achieve this density in the model, a coupling coefficient of $40 \%$ was

511 required, indicating the strength of DC-SIGN's attachment to the hypothesized directed

512 transport mechanism. While there is no direct measurement of this value for DC-SIGN

513 available at this time, this coupling coefficient is within the physiological range observed

514 in other systems involving receptor coupling to AMF[10,22]. This result suggests that

515 partial coupling of DC-SIGN to a centripetally directed active transport mechanism is a

516 viable model for explaining experimentally observable DC-SIGN recruitment to $C$.

517 albicans contact sites.

518 To determine the level of DC-SIGN recruitment that could be achieved in the

519 absence of directed motion, we ran simulations at a directed transport coupling coefficient

520 of zero, leaving only lateral mobility via Brownian diffusion (i.e., "Brownian" model). All

521 other parameters of the model were kept exactly the same as in the Directed + Brownian

522 model. We found that the Brownian model predicted contact site DC-SIGN density

523 increase of $54.02 \%$ (Figure 6). This is similar to what we observed in inhibitor conditions

524 which are supposed to inhibit directed transport of DC-SIGN at contact site (Figure 4). 


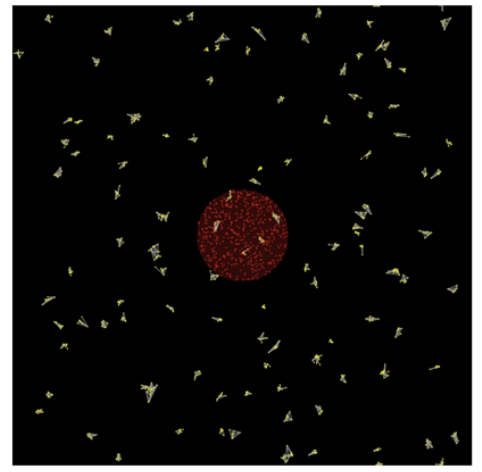

Pre-simulation conatct site

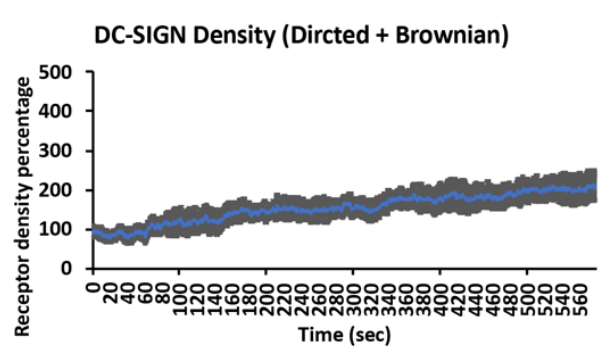

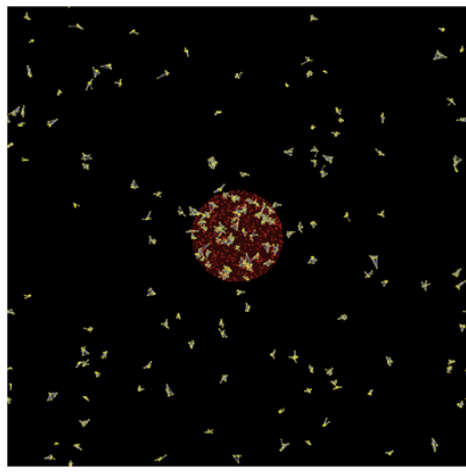

Post-simulation (Directed + Brownian)

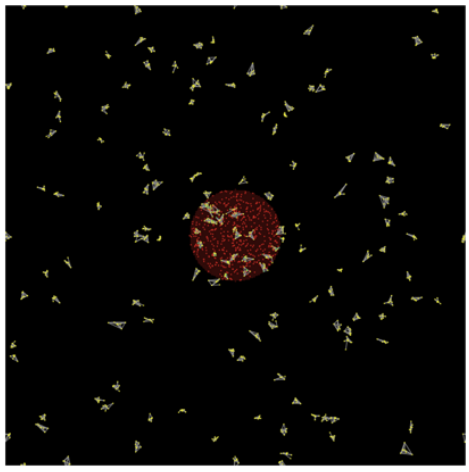

Post-simulation (Brownian only)

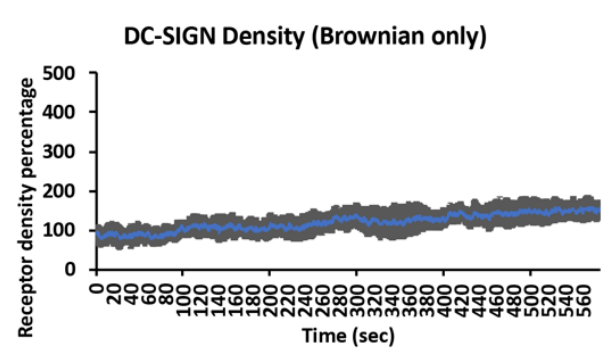

526 Figure 6. Agent-based modelling showing role of directed motion in DC-SIGN transport

527 at C. albicans contact sites. Top panels are views of DC-SIGN (yellow) distribution relative

528 to the contact site (red) before the start (top left) and after 10 minutes of simulation time

529 for models simulating Brownian diffusion only (top right) or directed transport

530 superimposed upon Brownian diffusion (Directed+Brownian) (top middle). Graphs

531 indicate the percentage increase in DC-SIGN density over pre-contact density within 10

532 minutes of simulation time for Brownian diffusion only model (bottom right) or

533 Directed+Brownian model (bottom left).

\section{4}

536 expected an enrichment of F-actin and DC-SIGN in these regions during the early stages

537 of contact site formation. TIRF microscopy is a useful approach with very high axial and

538 lateral resolution for visualizing protein recruitment dynamics at or very near the plasma 
539 membrane. However, TIRF microscopy requires the membrane region of interest to be

540 immediately adjacent to the cover glass - a condition not met in our previous contact site

541 experimental model. We therefore devised a contact site model amenable to TIRF

542 microscopy wherein HEK-293 cells transfected with EGFP-DC-SIGN, pUNO1-hDectin-

543 1a and mCardinal-LifeAct-7 were dropped onto $\beta$-glucan-coated cover glass surfaces. As

544 cells began to interact with this surface, they extended dynamic protrusive structures to

545 explore the glucan-coated glass. We found that DC-SIGN and F-actin (LifeAct) became

546 enriched within cell processes in contact with the glucan-coated surface, and that the

547 kinetics of this enrichment showed a temporal correlation of DC-SIGN and LifeAct

548 fluorescence, but with a lag of DC-SIGN relative to F-actin recruitment. The observed

549 latency of maximum DC-SIGN intensity relative to LifeAct was $8.35 \pm 4.57$ seconds. This

550 correlation was almost completely abolished by RHOA inhibitor, with DC-SIGN latency

551 of $1.69 \pm 4.97$ seconds in this condition (Figure S3), which was a statistically significant

552 decrease $(\mathrm{p}=0.024)$ relative to the non-inhibited condition. Thus giving direct imaging

553 based preliminary evidence for Dectin-1 mediated RHOA dependent actomyosin based

554 transport of DC-SIGN at C. albicans contact site.

\section{4. Discussion}

556 At $C$. albicans contacts with immune cells, which have been described as the

557 "phagocytic synapse", Goodridge et al. showed the accumulation of Dectin-1 within

558 contact sites between myeloid cell types and model fungal particles and fungal pathogen

559 cells wherein regulatory tyrosine phosphatases CD45 and CD148 were excluded from

560 Dectin-1 rich zones of the contact [7]. The evident recruitment of immunoreceptors at these

561 cellular synaptic structures and alterations to their patterns of lateral mobility in the 
562 membrane suggest that it is important to understand the molecular mechanisms responsible

563 for their construction. In the case of fungal host-pathogen contacts, many studies have

564 examined receptor distribution at tens of minutes to hours due to the difficulty of achieving

565 precise control over contact site formation that is necessary for examining the earliest

566 stages of host-pathogen interaction. We have overcome that problem in this study by the

567 use of micropipette-micromanipulator based application of fungal particles to cells with

568 high spatiotemporal precision.

569 We looked at the dynamics of DC-SIGN and Dectin-1 recruitment at the $\leq 10$ -

570 minute time scale. We propose that events occurring at these earliest stages of contact site

571 formation are important to promote pathogen capture and to stabilize the phagocytic

572 synapse. DC-SIGN showed significant contact site recruitment within the first 10 min.,

573 whereas Dectin-1 did not show recruitment within this period, relative to its initial density.

574 DC-SIGN is optimized for high avidity interactions with fungal pathogens due to its 575 tetramerization via its stalk domain and its organization into multi-tetramer

576 nanodomains[17,18,23]. Improvements in the efficiency of contact site recruitment of DC-

577 SIGN are likely to be important for determining the ability of the phagocytic synapse to

578 retain a fungal pathogen, especially under circumstances where fluid shear forces could

579 destabilize the contact site before internalization of the particle can take place.

580 In the absence of Dectin-1, there was a significant decrease in the recruitment of

581 DC-SIGN. In recently published work, we showed that Dectin-1 stimulation by $\beta$-glucan

582 gives rise to RHOA-mediated actomyosin activation for contractile mechanical force

583 generation. Previous research on the immunological synapse has demonstrated the

584 importance of RHO-GTPase mediated actin cytoskeleton organization in adhesion and 
585 early immunological synapse formation[24]. Further, the work of Tsourkas et al. with B

586 cell synapses showed with stochastic simulations that the formation of the synapse occurs

587 only if BCR mobility is enhanced by directed motor-driven transport [25]. Also, Manzo et

588 al. showed the role of lateral mobility of DC-SIGN nanoclusters in enhancing pathogen

589 binding using Monte Carlo simulations [18]. Because Dectin-1 activation appeared to

590 enhance DC-SIGN recruitment, we tested the role of RHOA-mediated actomyosin

591 activation downstream of Dectin-1, leading to active recruitment of DC-SIGN to $C$.

592 albicans contact sites. Using agent-based modeling, we found that a directed transport

593 process for DC-SIGN recruitment was necessary in order for computational predictions of

594 DC-SIGN recruitment kinetics to match experimentally observed rates of DC-SIGN

595 recruitment to host-pathogen contact sites. To further support this finding experimentally,

596 we found that inhibitors of RHOA, ROCK and Myosin II decreased DC-SIGN recruitment

597 to contact sites (Figure 4). The density of contact site DC-SIGN achieved in the presence

598 these inhibitors was similar to the DC-SIGN density predicted by computational modeling

599 of contact site biogenesis under the assumption that DC-SIGN was only transported by

600 passive diffusion followed by trapping in the contact via high avidity interactions with cell

601 wall N-mannans (Figure 6). This result strongly suggests that RHOA, ROCK and Myosin

602 II are essential components of a Dectin-1 dependent active transport mechanism that

603 enhances DC-SIGN recruitment to the sites of host-pathogen interaction within minutes of

604 pathogen contact.

605 RHOA and ROCK inhibited contacts exhibited a larger total area than without

606 inhibitor. We expect that the spreading of a cell over a pathogen surface to form a contact

607 site is a net result of protrusive (e.g., RAC1-mediated branched actin in lamellar edges and 
608 membrane ruffles) and contractile processes (i.e., RHOA-mediated actomyosin contraction

609 around the contact), both of which are unfolding over the time scale of our studies. The

610 fact that RHOA pathway inhibitors allow greater cell spreading to form larger area contacts

611 is compelling evidence that contractile mechanical forces are being generated during the

612 first ten minutes of host-pathogen interaction with C. albicans.

613 We did not find significant enhancement of Dectin-1 density in contact sites relative

614 to non-contact membrane within the first $10 \mathrm{~min}$. of C. albicans contact. We think this is

615 because sites of nanoscopic exposures of $\beta$-glucan on the $C$. albicans wall surface are

616 limited in spatial extent and sparsely distributed. As described by Ostrowski et al. for

617 phagocytic synapse, upon initial clustering of cognate receptors the ligands on the pathogen

618 surface trigger the signaling pathways that could initiate signaling and phagocytosis[8].

619 However, receptor density enrichment and continuous receptor-ligand interaction is

620 required to complete internalization of phagocytic particle, otherwise phagocytosis will

621 stall[26]. Our results are consistent with a model of C. albicans recognition wherein initial

622 Brownian diffusion of Dectin-1 leads to rapid Dectin-1 engagement and RHOA-mediated

623 actomyosin flow formation (Figure 6). Subsequently, DC-SIGN gets coupled to this

624 actomyosin flow, which facilitates its efficient mobilization to the contact site. Soon after

625 being drawn into the contact site, DC-SIGN nanodomains engage in high avidity

626 interactions with the fungal cell wall surface, which is important for strong retention of the

627 fungal particle. The high fraction of pathogen-interacting DC-SIGN in the contact site is

628 evident from our FRAP results where the majority of DC-SIGN is immobile over the time

629 scale of minutes (Figure 5). It is this prolonged pathogen retention that allows Dectin-1,

630 the pro-phagocytic receptor, to engage in the more time-consuming process of searching 
631 for and integrating signaling from sparse sites of nanoscale glucan exposure. This is evident

632 from a large mobile fraction of Dectin-1 in contacts sites at $10 \mathrm{~min}$. At larger time scales,

633 Dectin-1 may show significant enrichment within phagocytic contacts to amplify its

634 signaling ultimately giving rise to phagocytosis. This is supported by Tam et al. showing

635 accumulation of Dectin-1 within phagocytic synapse with glucan coated particles at 30

636 min. time scale[6]. Also, Strijibis et al. showed importance BTK, VAV1 and F-actin

637 accumulation within C. albicans contact site for efficient phagocytosis of fungus at an hour

638 scale[5]. Our results on the earliest stages of innate immune fungal recognition provide

639 some increased insight into host-pathogen contact site evolution, which is evidently a

640 complex, orchestrated process that involves many receptors being are recruited and

641 activated across different time scales.

642 Yi et al. showed direct evidence for actin retrograde flow and actomyosin II arc

643 contractions playing role in driving TCR cluster at T-cell immunologic synapse[10]. We

644 hypothesize that DC-SIGN can get coupled to a similar actomyosin flow in the phagocytic

645 synapse, leading to its early recruitment at (Figure 7). 


\section{Large recruitment of DC-SIGN at contact site beacuse of it's tetravalent interaction with abundnat mannan to stabilize contact site}

Initial Dectin-1 activation

\section{7}

648 Figure 7. Model for early recruitment of DC-SIGN at fungal contact site. Initial Dectin-1

649 activation leads to RHOA mediated actomyosin flow formation. DC-SIGN gets coupled to

650 this AMF and start accumulating within contact site, leading to stable capture of fungal

651 particles. After this early stabilization of contact site, Dectin-1 will get time to look for its

652 rare ligand to amplify its signaling, ultimately leading phagocytosis. (Figure created using

653 Biorender)

In contrast to our model, Liu et al. showed constitutive role of microtubule based

655 retrograde transport of DC-SIGN nanoclusters to bring pathogens to the perinuclear region

656 for antigen processing[21]. In this study, DC-SIGN nanoclusters were unladen with 
657 pathogen or attached to viral particles. It is possible that microtubule associated transport

658 is a constitutive retrograde transport process involved in receptor recycling or antigen

659 acquisition, but the conditions in Liu et al. did not involve AMF generation because Dectin-

660 1/RHOA axis signaling was absent. Nevertheless, future studies could examine potential

661 the contribution of microtubule mediated transport of DC-SIGN to nascent fungal contacts.

662 Cambi et al. discuss the possible role of DC-SIGN in direct phagocytosis of C. albicans by

663 immature dendritic cells [4]. They show enrichment of DC-SIGN within phagosome. This

664 finding is consistent with DC-SIGN's prominent role in capturing fungal particles, though

665 it also raises possible pro-phagocytic role of DC-SIGN. However, Rosa et al. showed that

666 DC-SIGN plays a role in binding of zymosan particles but is uninvolved in coordinating

667 phagocytic signaling of those particles. Consistent with our findings, Rosa et al. also

668 highlighted the prominent co-localization of actin and Dectin-1 within zymosan contact

669 site, as would be expected for Dectin-1 mediated actomyosin reorganization at the contact

670 site[19].

671 Geijtenbeek et al. and van Gisbergen et al. showed that DC-SIGN plays a role in

672 intercellular adhesion of DCs with T cells [27,28]. Using an assay of rapid cellular capture

673 of yeast under fluid shear conditions, we found that when Dectin-1 is co-expressed with

674 DC-SIGN, cells could capture significantly more fungal particles than when any of these

675 receptors are expressed individually. Thus, we concluded that the interplay between

676 Dectin-1 and DC-SIGN is important for optimal fungal capture and retention in early

677 fungal phagocytic contacts. ALS5 is a fungal amyloid mannoprotein adhesin which

678 undergoes reorganization into nanodomains under shear. This reorganization of ALS5

679 under shear exposes binding sites for DC-SIGN, thus making fungal particle sticky for DC- 
680 SIGN binding. Thus, it is possible that early, large recruitment of DC-SIGN will improve

681 avidity of interaction with Candida through DC-SIGN-ALS5 interactions (or other

682 mannoprotein adhesins) under flow conditions [29], which could be pursued in future

683 research.

684 In conclusion, we showed that Dectin-1 mediated activation of RHOA-ROCK-

685 Myosin II axis plays important role in active recruitment of DC-SIGN to the C. albicans

686 contact site. This is important for capture of fungal particles and the formation of stable

687 host-pathogen contact sites.

688

689 Acknowledgement

690 We gratefully acknowledge technical advice by Akram Etemadi Amin, Carmen

691 Martinez, Eduardo Anaya and Matthew Graus. The authors declare that they have no

692 conflicts of interest relevant to this work. This research was supported by the University of

693 New Mexico Center for Spatiotemporal Modeling of Cell Signaling (STMC; NIH

694 P50GM085273, AKN) and R01AI116894 (AKN).

695

696

697

698

699

700

701 


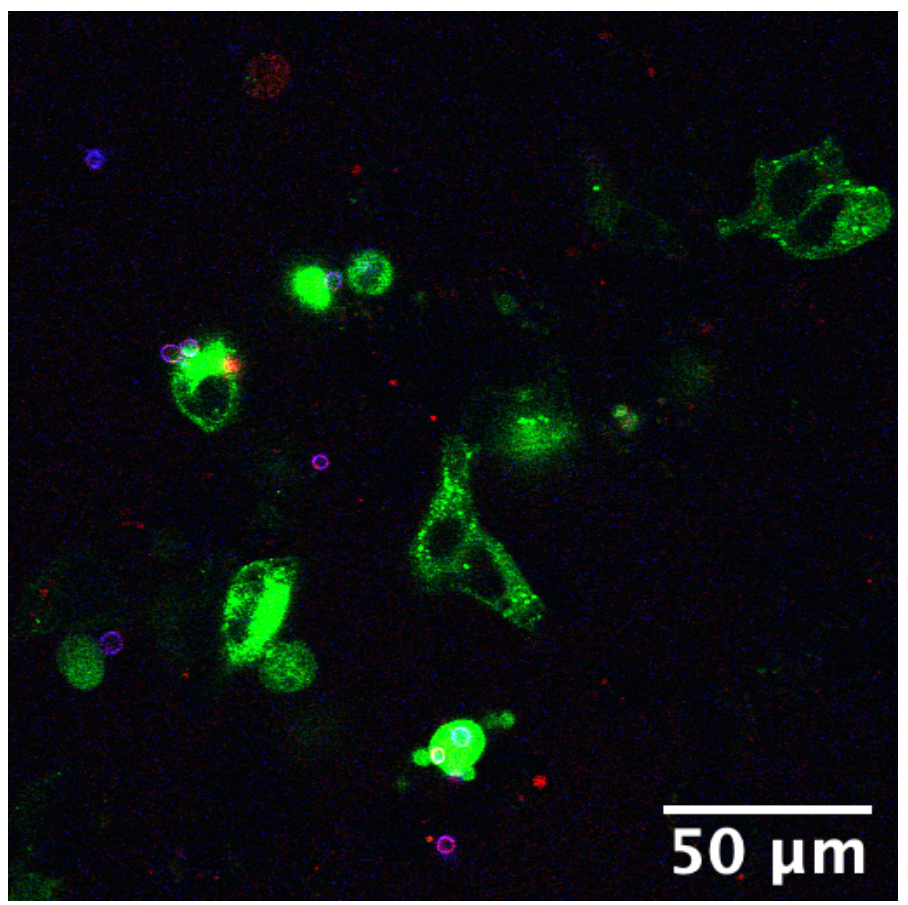

705 Figure S1. Phagocytosis assay in HEK-293 cells expressing EGFP-DC-SIGN (green) with

706 TRL035 C. albicans (blue, calcofluor, cell wall stain; red, cypher5e, pH sensitive

707 phagocytosis indicator). There was no uptake of fungal particles in the DC-SIGN only

708 condition.

709

710 

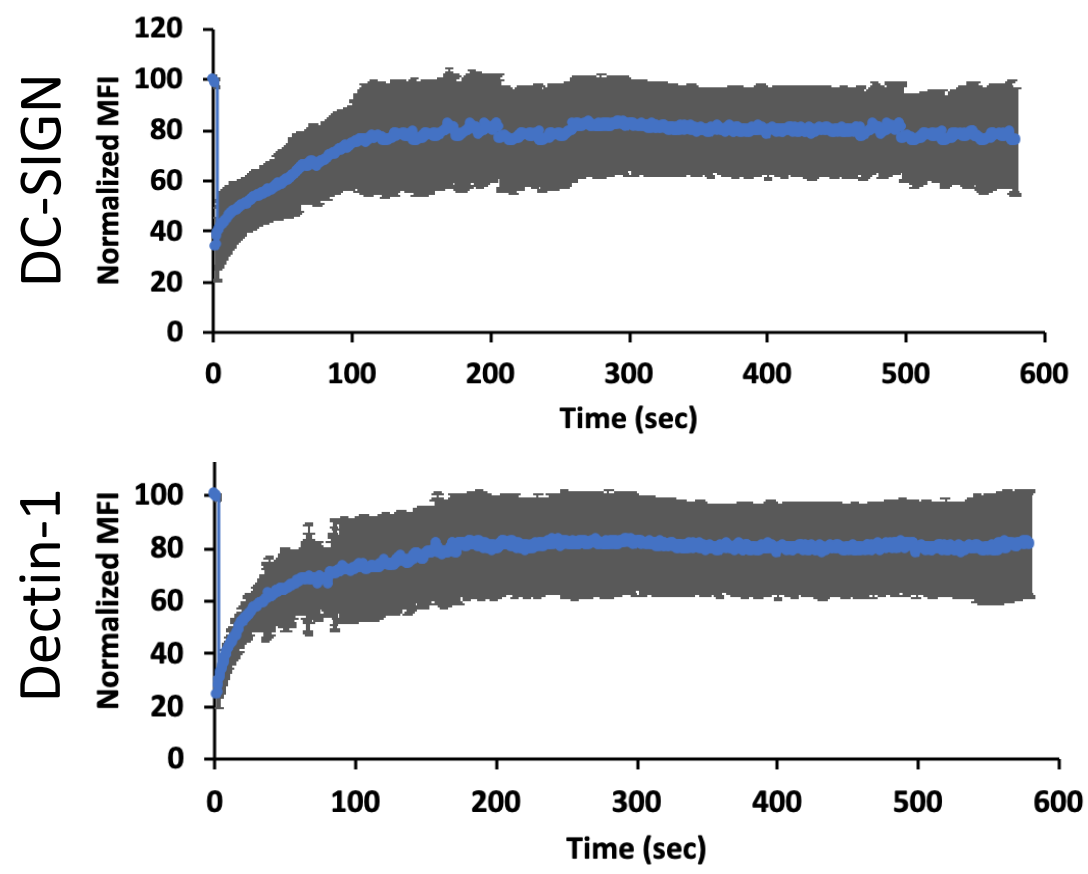

714 Figure S2. Effect of RHOA inhibitor on DC-SIGN and Dectin-1 mobility in non-contact

715 membranes. (top) DC-SIGN showed $75.72 \%$ mobile fraction and recovery $\mathrm{t}_{1 / 2}$ of $35.47 \mathrm{sec}$.

716 (bottom) Dectin-1 showed $81.77 \%$ mobile fraction and recovery $\mathrm{t}_{1 / 2}$ of $14.42 \mathrm{sec}$ 


\section{Latency of DC-SIGN fluroscence compared to Actin}

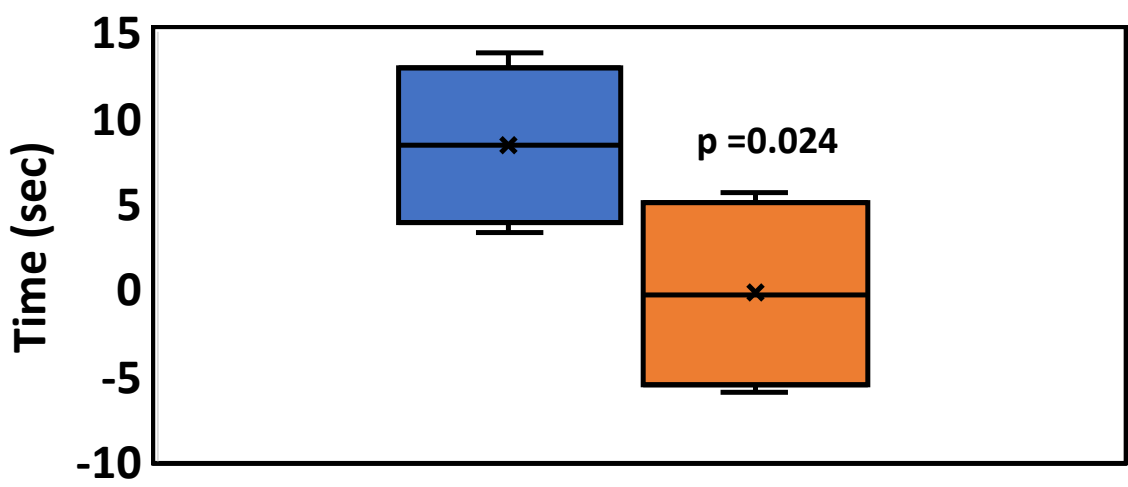

\section{口No inhibitor $\square$ RHOA inhibitor}

720 Figure S3. Latency for DC-SIGN compared to F-actin in nascent contact site membranes

721 for no inhibitor and RHOA inhibitor conditions. Box and whisker plot depicts mean ("x"),

722 median (horizontal bar within box), $1^{\text {st }}$ and $3^{\text {rd }}$ quartile (top and bottom of colored box) and

723 minimum and maximum value (whiskers). 


\section{Agent-based modelling: Detailed Methods}

727 For modeling transport of DC-SIGN to fungal contact sites we used Netlogo

728 version 6.1.1. The goal of the simulation was to look at the effects of various modes of

729 lateral transport of DC-SIGN on the kinetics of DC-SIGN recruitment at contact site. By

730 arbitrary convention, we kept world square with the dimension of $5 \mu \mathrm{m} \times 5 \mu \mathrm{m}$. All other

731 parameters were derived from previously available literature or systematically varied. The

732 essential script for the model is provide below.

733

734 Model Parameterization

735 Parameters which were kept constant:

736 - Contact site diameter was set at $1 \mu \mathrm{m}$ based on a measured value reported

737 previously[9].

738 - $1.7 \times 10^{-4} / \mathrm{nm}^{2}$ mannan binding sites were distributed randomly throughout contact

739 site, based on an estimation of a prominent C. albicans mannoprotein previous ly

$740 \quad$ reported [29].

741 - 2 DC-SIGN tetramers per domain were randomly distributed at domain density of

$742 \quad 1.2 \times 10^{-6} / \mathrm{nm}^{2}$ throughout world area based on prior optical nanoscopy

743 measurements $[17,18,23]$.

744 - Size of DC-SIGN nanodomain in resting membrane was approximated to $75 \mathrm{~nm}$

745 based on prior optical nanoscopy measurements [17].

746

747 Parameters which were varied in model: 
748 - For association and dissociation probability, we ran a separate simulation with single DC-SIGN CRD domains with single mannan moieties available for binding in the world. We optimized model for total simulation time, so that quantity of free and bound fraction of receptor and ligand is stabilized at the end of simulation. We could achieve equilibrium binding for all conditions in 1 hour of simulated time. So, we let model run for each combination of association and dissociation probabilities for 1 hour. Then we calculated $\mathrm{K}_{\mathrm{d}}$ for each condition using the quantity of unbound ligand, unbound receptor and receptor-ligand concentration. Previous literature has reported experimentally calculated $\mathrm{K}_{\mathrm{d}}$ for DC-SIGN and mannan interaction to be $50 \mu \mathrm{M}[30]$. Hence, we used association and dissociation probabilities which gave $\mathrm{K}_{\mathrm{d}}$ value between 30 to $60 \mu \mathrm{M}$ for contact site simulation, bracketing this value. $K_{d}$ values in this range are highlighted as red text in Table 2. Actual values used in simulations for data generation are detailed further below.

\begin{tabular}{|r|r|r|r|r|r|}
\cline { 2 - 7 } & Dissoc_prob & & & & \\
\hline Assoc_prob & $\mathbf{0 . 0 1}$ & $\mathbf{0 . 0 2}$ & $\mathbf{0 . 0 3}$ & $\mathbf{0 . 0 4}$ & $\mathbf{0 . 0 5}$ \\
\cline { 2 - 7 } & $5.22 \mathrm{E}-05$ & $9.08 \mathrm{E}-05$ & $1.52 \mathrm{E}-04$ & $1.82 \mathrm{E}-04$ & $1.52 \mathrm{E}-04$ \\
$\mathbf{0 . 3}$ & $2.18 \mathrm{E}-05$ & $4.00 \mathrm{E}-05$ & $1.01 \mathrm{E}-04$ & $1.11 \mathrm{E}-04$ & $1.21 \mathrm{E}-04$ \\
$\mathbf{0 . 3 5}$ & $1.83 \mathrm{E}-05$ & $3.66 \mathrm{E}-05$ & $1.03 \mathrm{E}-04$ & $9.28 \mathrm{E}-05$ & $9.95 \mathrm{E}-05$ \\
$\mathbf{0 . 4}$ & $2.39 \mathrm{E}-05$ & $3.19 \mathrm{E}-05$ & $8.57 \mathrm{E}-05$ & $1.03 \mathrm{E}-04$ & $7.55 \mathrm{E}-05$ \\
$\mathbf{0 . 4 5}$ & $1.40 \mathrm{E}-05$ & $3.39 \mathrm{E}-05$ & $6.54 \mathrm{E}-05$ & $1.11 \mathrm{E}-04$ & $1.11 \mathrm{E}-04$ \\
$\mathbf{0 . 5}$ & 1.04 & $1.37 \mathrm{E}-04$ & $1.11 \mathrm{E}-04$ \\
$\mathbf{0 . 5 5}$ & $1.79 \mathrm{E}-05$ & $3.74 \mathrm{E}-05$ & $6.12 \mathrm{E}-05$ & 1.35 & $1.06 \mathrm{E}-04$ \\
$\mathbf{0 . 6}$ & $1.52 \mathrm{E}-05$ & $2.31 \mathrm{E}-05$ & $5.01 \mathrm{E}-05$ & $6.03 \mathrm{E}-05$ & $1.52 \mathrm{E}-04$ \\
\hline $\mathbf{0 . 6 5}$ & $1.09 \mathrm{E}-05$ & $2.59 \mathrm{E}-05$ & $4.71 \mathrm{E}-05$ & $4.87 \mathrm{E}-05$ & 1.05 \\
\hline $\mathbf{0 . 7}$ & $1.71 \mathrm{E}-05$ & $2.33 \mathrm{E}-05$ & $4.51 \mathrm{E}-05$ & $3.69 \mathrm{E}-05$ & $5.01 \mathrm{E}-05$ \\
\hline & & & & & \\
\hline Green $<3 \mathrm{E}-05$ & & Red 3E-05 to 6E-05 & Yellow $>6 \mathrm{E}-05$ & \\
\hline
\end{tabular}

Table 2. $K_{d}$ value obtained after simulation of each condition of association and dissociation probability for 1 hour. Each condition was simulated in triplicate. Red 
indicate values around experimentally calculated $\mathrm{K}_{\mathrm{d}}$ of DC-SIGN-Mannan interaction.

- Probability for DC-SIGN carbohydrate recognition domain to couple (coupling coefficient) to actomyosin flow was varied between 0 to $80 \%$. This was from previously available literature for immunologic synapse [10,22].

771 - Step sigma for DC-SIGN is based on diffusion coefficient value of $6.5 \times 10^{-2}$ $772 \mu \mathrm{m}^{2} / \mathrm{sec}$ for DC-SIGN domain reported by Manzo et al.[18] The mean diffusion 773 radius was obtained from

$774 \mathrm{r}^{2}=4 \mathrm{Dt}\left(\mathrm{D}=6.5 \times 10^{-2} \mu \mathrm{m}^{2} / \mathrm{sec}, \mathrm{t}=0.1 \mathrm{~s}\right.$ time of a clock tick for model $)$

$775 \quad$ Hence, $\mathrm{r}=161 \mathrm{~nm}$. $\mathrm{nm}$. However, Manzo et al. also measured that $10 \%$ of trajectories are immobile, so we modeled this as DC-SIGN domain movements with a $90 \%$ probability[18]. derived from previously reported values for immune synapse[10,31]. motion of $0.10 \mu \mathrm{m} / \mathrm{sec}$, fixed heading towards to center of contact site with $x \%$ probability each time step. random heading and $161 \mathrm{~nm}$ diffusion radius in each time step. 

wrapping of world. Also, to compensate for DC-SIGN which were bound and inside contact site, we replenished the equivalent number of DC-SIGNs to the outside world. This was necessary it would have been computationally infeasible to model the whole cell's membrane, so we just simulated a small membrane patch. This patch (within the model and outside the contact site zone) is assumed to be freely connected by diffusion to the rest of the cell, which provides an effectively infinite pool of receptor in diffusive equilibrium with the modeled membrane patch. So, we assumed that the concentration of receptor outside contact area should always approximate the entire cell. Even if receptor is lost to the contact site membrane, this would have a negligible impact on the average concentration of receptor in membrane outside the simulation area. probability. So, if DC-SIGN is within binding radius of unbound mannan binding site then probability that it will bind to mannan binding site was as per association probability. Also, bound DC-SIGN dissociated from mannan binding site as per 804 dissociation probability.

- Model itself was run for $10 \mathrm{~min}$. for each condition. This is to keep simulation conditions same as actual experimental conditions. 
811 simulation runs (at a given parameter set) and the single simulation replicate's result was

812 considered as the mean behavior of those individual runs. Table 3 reports the mean of these

813 triplicates' results.

\begin{tabular}{|l|r|r|r|r|r|r|r|r|r|r|r|r|}
\hline Dissoc prob & \multicolumn{1}{|c|}{0.01} & \multicolumn{3}{|c|}{0.02} & \multicolumn{3}{|c|}{0.03} & \multicolumn{2}{|c|}{04} & 0.05 \\
\hline Assoc proba & 0.3 & 0.35 & 0.4 & 0.45 & 0.5 & 0.55 & 0.6 & 0.65 & 0.7 & 0.65 & 0.7 & 0.7 \\
\hline Coupling & & & & & & & & & & & & \\
\hline 0 & 93.95 & 9.83 & 42.29 & 49.71 & 54.02 & 60.59 & 39.17 & 72.3 & -54.43 & -3.52 & 29.9 & 11.1 \\
\hline 20 & 173.18 & 31.74 & 64.63 & 72.24 & 82.46 & 128.92 & 45.02 & 90.57 & 27.5 & 18.59 & 46.96 & 62.46 \\
\hline 40 & 214.93 & 45.4 & 79.51 & 85.56 & 108.72 & 157.02 & 59.58 & 94.86 & 45.59 & 32.59 & 49.39 & 128.92 \\
\hline 60 & 366.76 & 173.03 & 170.09 & 181.73 & 212.45 & 260.17 & 182.88 & 185.55 & 125.56 & 72.48 & 144.37 & 270.28 \\
\hline 80 & 646.54 & 552.1 & 367.13 & 221.61 & 391.75 & 486.3 & 238.41 & 284.37 & 326.79 & 158.19 & 255.02 & 196.8 \\
\hline
\end{tabular}

815 Table 3. Final increase in DC-SIGN densities compared to pre-contact densities at the end

816 of $10 \mathrm{~min}$. simulation for each specific condition of association probability, dissociation

817 probability and coupling coefficient. Each condition was simulated in triplicate.

We found that dissociation probability of 0.02 and association probability of 0.5

821 in table 3.3), this is similar to the experimental value of $103.15 \%$ obtained with DC-SIGN

822 and Dectin-1 co-expression contact sites (Fig. 3.1e).

824 of 0.5 with 0 coupling gave increase in DC-SIGN density of 54.02\% (highlighted in gray

825 in table 3.3). This is similar to what we obtained with RHOA, ROCK and Myosin-II

826 inhibitors which showed DC-SIGN recruitment of $41.47 \%, 62.96 \%$ and $44.04 \%$

827 respectively (Fig. 3.3).

828 Overall, in table only dissociation probability of 0.02 and association probability of

8290.5 gave a global best fit to what we observed experimentally. 
832 ;Simulation of DCSIGN domains on a DC interacting with a fungal contact site 833 displaying Als family adhesins.

834 ;The goal of this simulation is to investigate the effects of binding avidity and

835 mode of lateral transport

836 ;on contact site structures formed by DCSIGN and the kinetics of DCSIGN

837 recruitment.

838 ;Aaron Neumann Mar 082014

839 ;updated by Rohan Choraghe May 202020

840

841

842

843

844

845

846

847

848

849

850

851

852

globals

853

854

855

856

857

858

859

860

861

862

[

domain_sigma_m

step_sigma_m

contact_radius_m

binding_radius_m

domain_x

domain_y

domain_heading

next_heading

next fd

domain_counter

domain_create_counter

domain_number

density_setpt

863

864

865

DCSIGN_deficit

DCSIGN_domain_needed

contact_fraction ; the fraction of DCSIGN domains in the contact

density_enrichment ;the fold amount of DCSIGN domains in the contact

relative to what would be expected by random distribution in the ;contact site's area.

radial_shell_domains; counts the number of DCSIGN domains in the outer $10 \%$ radial shell of the contact.

filename_view filename_world

866 AMF $m$

867 density_enrichment_2

868

869

870

]

coupling_random

871

872

873

breed [DCSIGNs DCSIGN]

DCSIGNs-own [domain_ID bound which_ALS_bound]

874

875

876 to setup

877 clear-all 
878

879

880

881

882

883

884

885

886

887

888

889

890

891

892

893

894

895

896

897

898

899

900

901

902

903

904

905

906

907

908

909

910

911

912

913

914

915

916

917

918

919

920

921

922

923

reset-ticks

;assigns key variables that control domain formation and mobility set domain_sigma_m domain_sigma / patch_size ;size of DCSIGN domains set AMF_m AMF / (patch_size * tick_per_sec) ; how far DCSIGN moves with AFM set step_sigma_m step_sigma / patch_size ;how far DCSIGN domain moves per time tick set domain_number int (domain_density * (world-width * world-height * patch_size $\left.{ }^{\wedge}-2\right)$; ;how many DCSIG set contact_radius_m contact_radius / patch_size ;size of contact site between DC and yeast set binding_radius_m binding_radius / patch_size ;size of zone within which binding can happen

set filename_view word (word (word (word "Run\#" behaviorspace-run-number ) "_" ) date-and-time ) ".png"

set filename_world word (word (word (word "Run\#" behaviorspace-run-number ) "_ ) date-and-time ) ".csv"

;creates domain groups, spreads in gaussian shape of domain, forms links to other members of domain set domain_create_counter 1 repeat domain_number<smiles>CCCCCC[Tl]</smiles>

set domain_x random-xcor

set domain_y random-ycor

create-DCSIGNs DCSIGNs_per_domain * DCSIGN_multimerization [

set color yellow

set bound false

set which_ALS_bound -1

setxy domain_xdomain_y

set heading random 360

fd random-normal 0.0 domain_sigma_m

set domain_ID domain_create_counter

ask one-of $\bar{D}$ CSIGNs with [domain_ID = domain_create_counter]

[ create-links-with other DCSIGNs with [domain_ID =

domain_create_counter]

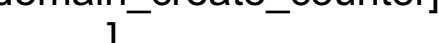


924 ;creates ALS proteins in random distribution and initializes them for gyration

925 mobility

926

927

928

929 set color red

create-ALSs int $\left(A L S \_d e n s i t y{ }^{*}\right.$ pi $^{*}$ contact_radius $\left.{ }^{\wedge} 2\right)$

930 set bound false

931

932

933

934

935

936

937

938

939

940

941

942

943

944

945 set which_DCSIGN_bound -1

set xcor (random-float $\left(2{ }^{*}\right.$ contact_radius_m) - contact_radius_m) set ycor (random-float $\left(2{ }^{*}\right.$ contact_radius_m) - contact_radius_m) while [ $\operatorname{sqrt}\left(\operatorname{xcor}^{\wedge} 2+\operatorname{ycor}^{\wedge} 2\right)>$ contact_radius_m] $^{\wedge}$

[

set xcor (random-float $(2$ * contact_radius_m) - contact_radius_m)

set ycor (random-float $(2$ * contact_radius_m) - contact_radius_m)

946

947

]

set orig_x xcor

set orig_y ycor

set ALS_gyration_radius_m ALS_gyration_radius / patch_size

948

949

]

950

;shades the patches in the contact site dark red

ask patches with [sqrt $\left((\text { pxcor * patch_size })^{\wedge} 2+\left(\text { pycor }^{*} \text { patch_size }\right)^{\wedge} 2\right)<=$ contact_radius] [set pcolor 11]

951

;defines the density setpoint that will be used to keep the non-contact area at constant DCSIGN density

;units: DCSIGN polypeptides per um² simulation space

952

set density_setpt domain_density *DCSIGNs_per_domain *

953

DCSIGN_multimerization

954

955

956

;reports the total sim area

957

type "Total simulation area $\left(u^{\wedge}{ }^{\wedge} 2\right)=$ " print $($ world-width * world-height *

958

patch_size ${ }^{\wedge}$ 2) / 1000000

959

960

961

end

962

963

964

965

966

967

968

;determines binding and immobilization of DCSIGNs and ALSs (probabilistic)

to go

969

ask DCSIGNs with [bound $=$ false AND count ALSs with [distance myself $<=$ binding_radius_m and bound $=$ false $]>0$ ] 


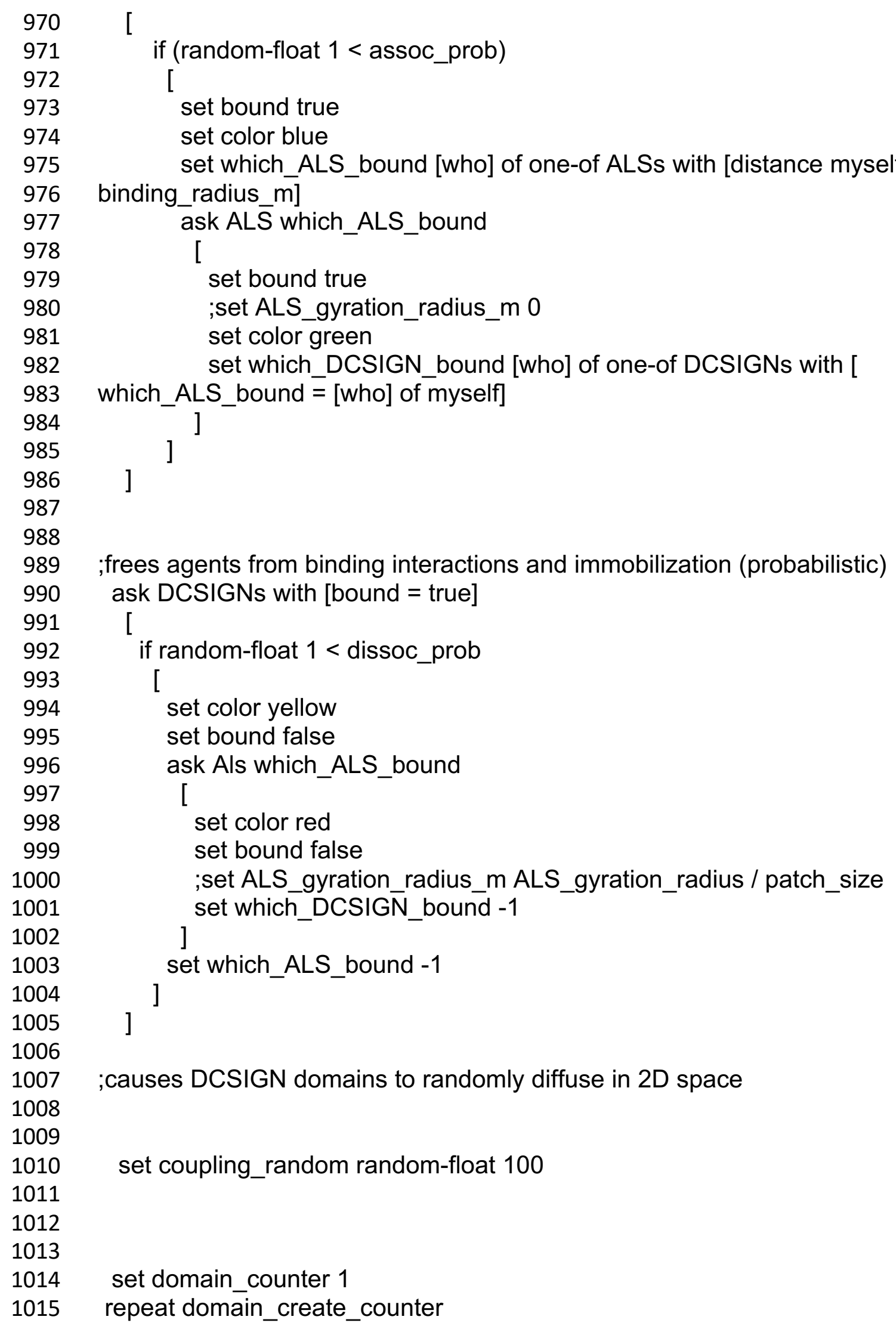

970

971

972

973

974

975

976

977

978

979

980

981

982

983

984

985

986

987

988

989

990

991

992

993

994

995

996

997

998

999

1000

1001

1002

1003

1004

1005

1006

1007

1008

1009

1010

1011

1012

1013

1014

1015 


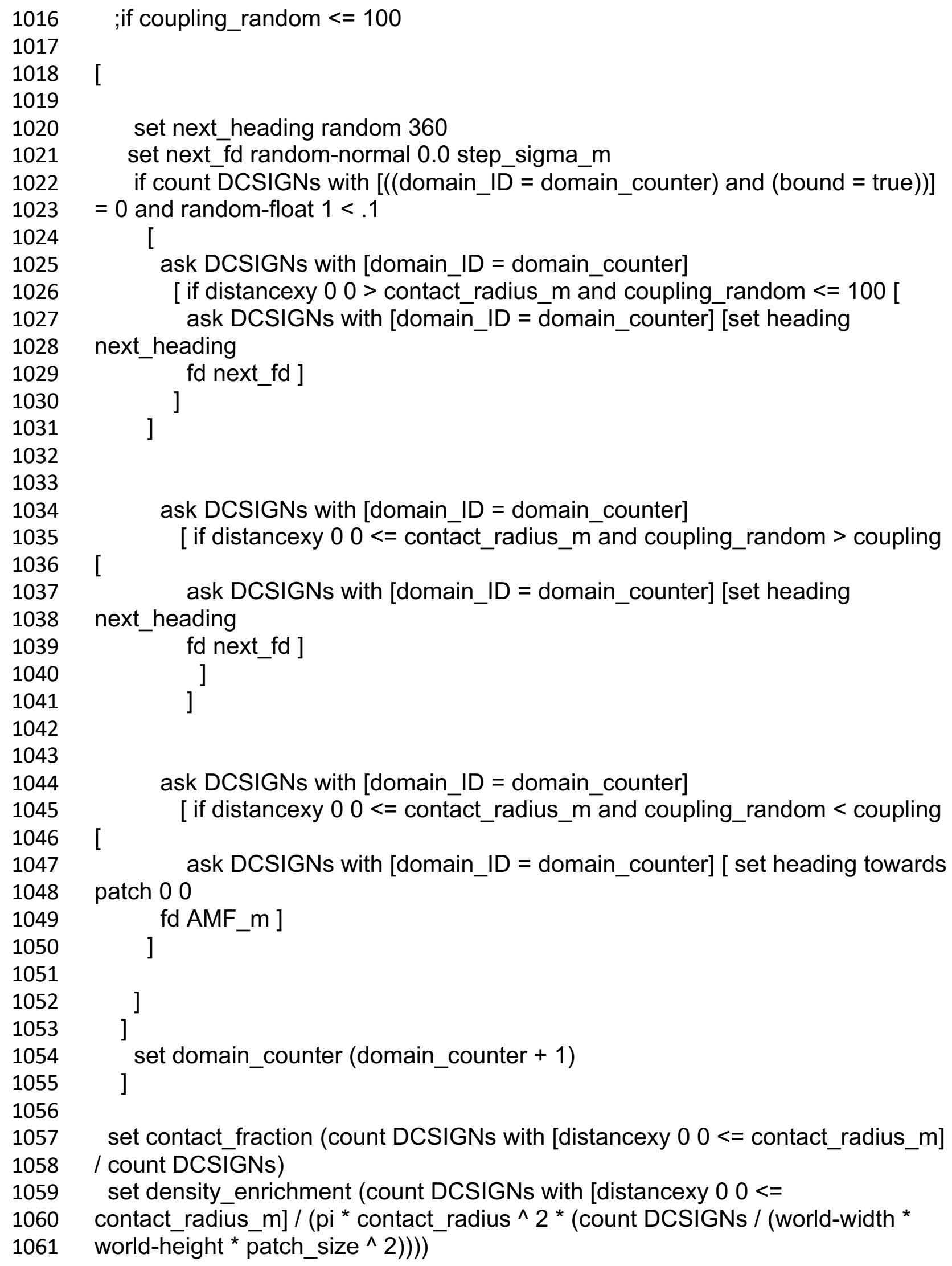


1062 set density_enrichment_2 (count DCSIGNs with [distancexy $00<=$

1063 contact_radius_m] / ( pi $^{*}$ contact_radius $\left.\left.{ }^{\wedge} 2\right)\right) /(($ count DCSIGNs - count

1064 DCSIGN̄s with [distancexy $00<=$ contact_radius_m]) / ((world-width * world-

1065 height * patch_size $\left.{ }^{\wedge} 2\right)-\left(\right.$ pi $^{*}$ contact_radius $\left.\left.\left.{ }^{\wedge} 2\right)\right)\right)^{-}$

1066

1067

set radial_shell_domains ((count DC SIGNs with [distancexy $00<=$

1068 contact_radius_m and distancexy $00>(0.9 *$ contact_radius_m $)])$ /

world-height * patch_size $\left.\left.{ }^{\wedge} 2\right)\right)-\left(\mathrm{pi}^{*}\left(0.9^{*}\right.\right.$ contact_radius $\left.^{\wedge} 2\right){ }^{*}($ count DCSIGNs

1071

1072

1073

1074

1075

1076

1077

1078

1079

1080

1081

1082

1083

1084

1085

1086

1087

1088

1089

1090

1091

1092

1093

1094

1095

1096

1097

1098

1099

1100

1101

1102

1103

1104

1105

1106

1107 / (world-width * world-height * patch_size $\left.\left.\left.\left.{ }^{\wedge} 2\right)\right)\right)\right)$ )

;create new DCSIGNs to keep the non-contact area at setpoint as they become trapped in the contact site

set DCSIGN_deficit (int (density_setpt * ((world-width * world-height * patch_size $\left.{ }^{\wedge} 2\right)-\left(\right.$ pi $^{*}$ contact_radius $\left.\left.{ }^{\wedge} 2\right)\right)$ -

(count DCSIGNs with [distancexy $00>$ contact_radius_m]) ))

;DCSIGN_domain_needed tells how many domains need to be created.

;Nothing should be created in the model until the deficit reaches the size of an entire domain

set DCSIGN_domain_needed int (DCSIGN_deficit / (DCSIGNs_per_domain * DCSIGN_multimerization))

repeat DCSIGN_domain_needed

[

set domain_heading random 360

create-DCSIGNs DCSIGNs_per_domain * DCSIGN_multimerization [

set color yellow

set bound false

set which_ALS_bound -1

set heading domain_heading

fd max-pxcor * 0.9

set heading random 360

fd random-normal 0.0 domain_sigma_m

set domain_ID domain_create_counter

ask one-of $\bar{D}$ CSIGNs with [domain_ID = domain_create_counter]

[

create-links-with other DCSIGNs with [domain_ID =

domain_create_counter]

]

]

set domain_create_counter (domain_create_counter + 1)

] 
end

1113 Model Script: Kd estimation

1114 ;Simulation of single DC-SIGN CRD domains interacting with single mannan moiety to estimate Kd for interaction.

1116 ;Aaron Neumann. Mar 082014

1117 ;edited by Rohan Choraghe April 042020.

1118

1119

1120

1121

1122

1123

1124

1125

1126

1127

1128

1129

1130

1131

1132

1133

1134

1135

1136

1137

1138

1139

globals

[

domain_sigma_m

step_sigma_m

binding_radius_m

domain_ $x$

domain_y

domain_heading

multimer_heading

next_heading

next_fd

domain_counter

domain_create_counter

domain_number

density_setpt

DCSIGN_deficit

DCSIGN_domain_needed

1140

1141

1142

1143

1144

1145

1146

1147

1148

1149

patch_volume ;this is the volume for 2D binding calcs assuming a membrane_separation distance

$\mathrm{R} L \mathrm{RL} \overline{\mathrm{Kd}}$; the apparent dissociation constant at a moment in time and vars

used to calc it. meant to be read out after the model has equilibrated

Kd_list Kd_list_avg

filename_view filename_world

]

breed [DCSIGNs DCSIGN]

DCSIGNs-own [domain_ID bound which_ALS_bound]

breed [ALSs ALS]

1150

ALSs-own [orig_x orig_y ALS_gyration_radius_m bound which_DCSIGN_bound]

1151 
1152 to setup

1153 clear-all

1154 reset-ticks

1155

1156

1157

1158

1159

1160

1161

1162

1163

1164

1165

1166

set ALS_density 4e-6

set DCSIGN_multimerization 1 set DCSIGNs_per_domain 1

set domain_density $4 \mathrm{e}-6$

set ALS_gyration_radius 50

set domain_sigmā 0

set step_sigma 161

set contact_radius 0

set binding_radius 5 ; dont change

set tick_per_sec 10 ; dont change

set patch_size 500 ; dont change

set membrane_separation 25 ; dont change

set Kd_list [0]; Needed to initialize the Kd_list so that it won't throw an error the first time.

set Kd_window 100

;assigns key variables that control domain formation and mobility set domain_sigma_m domain_sigma / patch_size ;size of DCSIGN domains set step_sigma_m step_sigma / patch_size ; per time tick

set domain_number int (domain_density * (world-width * world-height * patch_size $\left.{ }^{\wedge}{ }^{-2}\right)$ ) ; how many DCSIGN domains

set binding_radius_m binding_radius / patch_size ;size of zone within which binding can happen

set filename_view word (word (word (word "Run\#" behaviorspace-run-number ) "_" ) date-and-time ) ".png"

set filename_world word (word (word (word "Run\#" behaviorspace-run-number ) "_" ) date-and-time ) ".csv"

; creates domain groups, spreads in gaussian shape of domain, forms links to other members of domain

set domain_create_counter 1

repeat domain_number

[

1196

set domain_x random-xcor

1197 set domain_y random-ycor 
1198

1199

1200

1201

1202

1203

1204

1205

1206

1207

1208

1209

1210

1211

1212

1213

1214

1215

1216

1217

1218

1219

1220

1221

1222

1223

1224

1225

1226

1227

1228

1229

1230

1231

1232

1233

1234

1235

1236

1237

1238

1239

1240

1241

1242

1243

create-DCSIGNs DCSIGNs_per_domain * DCSIGN_multimerization

[

set color yellow

set bound false

set which_ALS_bound -1

setxy domain_xdomain_y

set heading random 360

fd random-normal 0.0 domain_sigma_m

set domain_ID domain_create_counter

]

set domain_create_counter (domain_create_counter + 1)

]

;creates ALS proteins in random distribution and initializes them for gyration

mobility

;Following creates the random distribution of ligand for $\mathrm{Kd}$ (non-contact) studies.

create-ALSs int (ALS_density * world-width * world-height * patch_size ${ }^{\wedge}$ 2)

[

set color red

set bound false

set which_DCSIGN_bound -1

set xcor random-xcor

set ycor random-ycor

set orig_x xcor

set orig_y ycor

set ALS_gyration_radius_m ALS_gyration_radius / patch_size

]

;reports the total sim area

type "Total simulation area $\left(\mathrm{um}^{\wedge} 2\right)=$ " print $($ world-width * world-height *

patch_size $^{\wedge}$ 2) / 1000000

end

to go

ask DCSIGNs with [bound = false AND count ALSs with [distance myself <= binding_radius_m] $>0$ ]

[

if (random-float $1<$ assoc_prob)

[

set bound true 
1244

1245

1246

1247

1248

1249

1250

1251

1252

1253

1254

1255

1256

1257

1258

1259

1260

1261

1262

1263

1264

1265

1266

1267

1268

1269

1270

1271

1272

1273

1274

1275

1276

1277

1278

1279

1280

1281

1282

1283

1284

1285

1286

1287

1288

1289 set color blue

set which_ALS_bound [who] of one-of ALSs with [distance myself <=

binding_radius_m]

ask ALS which_ALS_bound

[

set bound true

set ALS_gyration_radius_m 0

set color red

set which_DCSIGN_bound [who] of one-of DCSIGNs with [

which_ALS_bound $=$ [who] of myself]

;frees agents from binding interactions and immobilization (probabilistic)

ask DCSIGNs with [bound = true]

[

if random-float $1<$ dissoc_prob

[

set color yellow

set bound false

ask Als which_ALS_bound

[

set color red

set bound false

set ALS_gyration_radius_m ALS_gyration_radius / patch_size set which_DCSIGN_bound -1

]

set which_ALS_bound -1

]

]

set domain_counter 1

repeat domain_create_counter

[

set next_heading random 360

set next_fd random-normal 0.0 step_sigma_m

if count $\bar{D}$ CSIGNs with [((domain_ID $=$ domain_counter $)$ and (bound $=$ true $))]$

$=0$ and random-float $1<.1$

[

ask DCSIGNs with [domain_ID = domain_counter]

[

set heading next_heading 


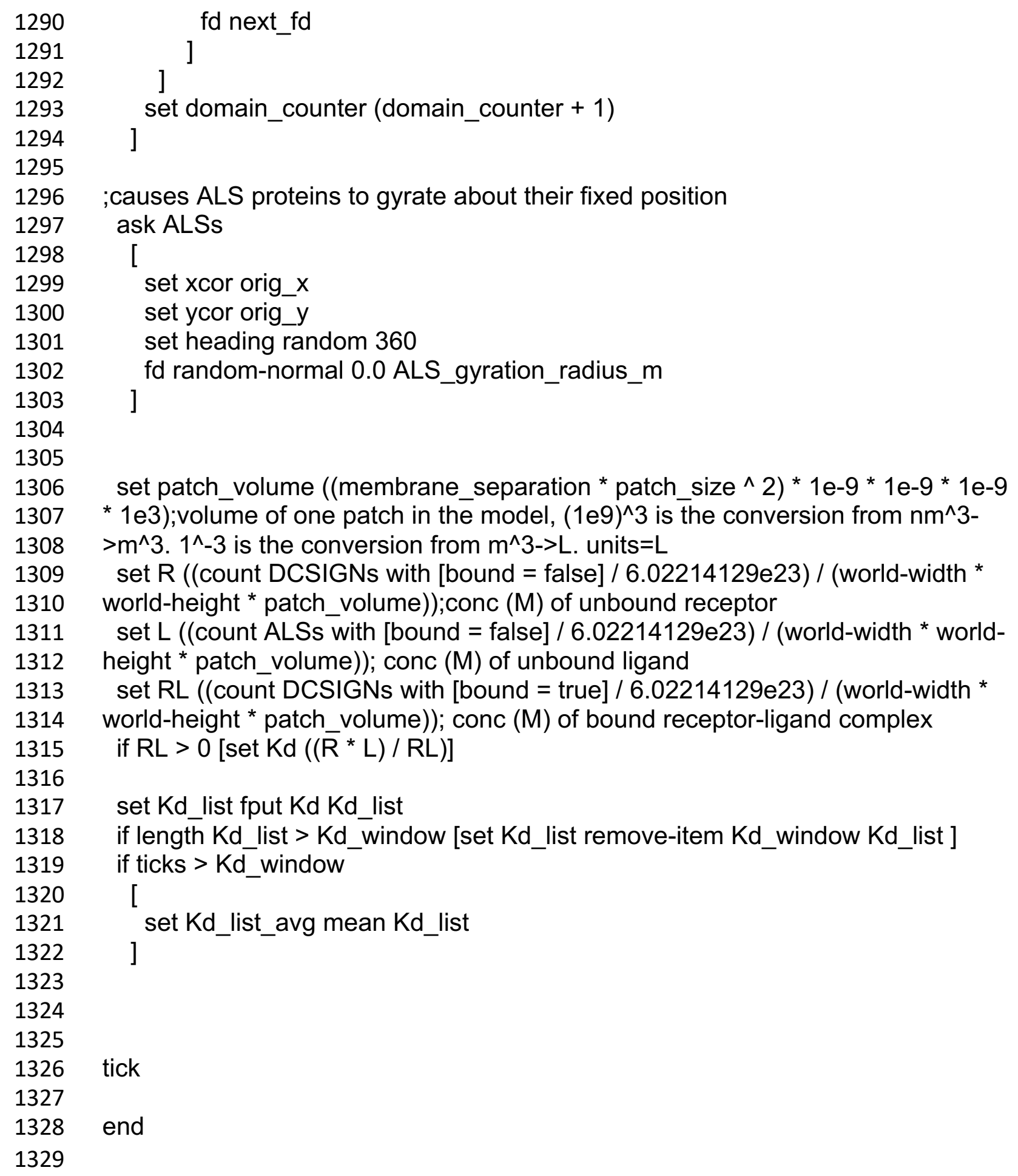




\section{References}

1332 1. Plato, A.; Willment, J.A.; Brown, G.D. C-Type Lectin-Like Receptors of the Dectin-1 Cluster: Ligands and Signaling Pathways. Int. Rev. Immunol. 2013, 32, 134-156, doi:10.3109/08830185.2013.777065.

2. Garcia-Vallejo, J.J.; van Kooyk, Y. The physiological role of DC-SIGN: A tale of mice and men. Trends Immunol. 2013, 34, 482-486, doi:10.1016/j.it.2013.03.001.

3. Neumann, A.K.; Jacobson, K. A Novel Pseudopodial Component of the Dendritic Cell Anti-Fungal Response: The Fungipod. PLoS Pathog. 2010, 6, e1000760, doi:10.1371/journal.ppat.1000760.

4. Cambi, A.; Gijzen, K.; Vries, I.J.M. de; Torensma, R.; Joosten, B.; Adema, G.J.; Netea, M.G.; Kullberg, B.-J.; Romani, L.; Figdor, C.G. The C-type lectin DC-SIGN (CD209) is an antigen-uptake receptor for Candida albicans on dendritic cells. Eur. J. Immunol. 2003, 33, 532-538, doi:10.1002/immu.200310029.

5. Strijbis, K.; Tafesse, F.G.; Fairn, G.D.; Witte, M.D.; Dougan, S.K.; Watson, N.; Spooner, E.; Esteban, A.; Vyas, V.K.; Fink, G.R.; et al. Bruton's Tyrosine Kinase (BTK) and Vav1 Contribute to Dectin1-Dependent Phagocytosis of Candida albicans in Macrophages. PLoS Pathog. 2013, 9, e1003446, doi:10.1371/journal.ppat.1003446.

6. Tam, J.M.; Mansour, M.K.; Khan, N.S.; Yoder, N.C.; Vyas, J.M. Use of fungal derived polysaccharide-conjugated particles to probe Dectin-1 responses in innate immunity. Integr Biol 2012, 4, 220-227, doi:10.1039/C2IB00089J.

7. Goodridge, H.S.; Reyes, C.N.; Becker, C.A.; Katsumoto, T.R.; Ma, J.; Wolf, A.J.; Bose, N.; Chan, A.S.H.; Magee, A.S.; Danielson, M.E.; et al. Activation of the innate immune receptor Dectin-1 upon formation of a 'phagocytic synapse.' Nature 2011, 472, 471-475, doi:10.1038/nature10071.

8. Ostrowski, P.P.; Grinstein, S.; Freeman, S.A. Diffusion Barriers, Mechanical Forces, and the Biophysics of Phagocytosis. Dev. Cell 2016, 38, 135-146, doi:10.1016/j.devcel.2016.06.023.

9. Graus, M.S.; Pehlke, C.; Wester, M.J.; Davidson, L.B.; Steinberg, S.L.; Neumann, A.K. A New Tool to Quantify Receptor Recruitment to Cell Contact Sites during Host-Pathogen Interaction. PLoS Comput. Biol. 2014, 10, e1003639, doi:10.1371/journal.pcbi.1003639.

10. Yi, J.; Wu, X.S.; Crites, T.; Hammer, J.A. Actin retrograde flow and actomyosin II arc contraction drive receptor cluster dynamics at the immunological synapse in Jurkat T cells. Mol. Biol. Cell 2012, 23, 834-852.

11. Choraghe, R.P.; Kołodziej, T.; Buser, A.; Rajfur, Z.; Neumann, A.K. RHOAmediated mechanical force generation through Dectin-1. J. Cell Sci. 2020, 133, jcs236166, doi:10.1242/jcs.236166.

12. Liu, P.; Wang, X.; Itano, M.S.; Neumann, A.K.; Jacobson, K.; Thompson, N.L. The Formation and Stability of DC-SIGN Microdomains Require its Extracellular Moiety. Traffic 2012, 13, 715-726, doi:10.1111/j.1600-0854.2012.01337.x.

13. Pappas, H.C.; Sylejmani, R.; Graus, M.S.; Donabedian, P.L.; Whitten, D.G.; Neumann, A.K. Antifungal Properties of Cationic Phenylene Ethynylenes and Their Impact on $\beta$-Glucan Exposure. Antimicrob. Agents Chemother. 2016, 60, 45194529, doi:10.1128/AAC.00317-16. 
1376

1377

1378

1379

1380

1381

1382

1383

1384

1385

1386

1387

1388

1389

1390

1391

1392

1393

1394

1395

1396

1397

1398

1399

1400

1401

1402

1403

1404

1405

1406

1407

1408

1409

1410

1411

1412

1413

1414

1415

1416

1417

1418

1419

1420

1421
14. Koulouras, G.; Panagopoulos, A.; Rapsomaniki, M.A.; Giakoumakis, N.N.; Taraviras, S.; Lygerou, Z. EasyFRAP-web: a web-based tool for the analysis of fluorescence recovery after photobleaching data. Nucleic Acids Res. 2018, 46, W467-W472, doi:10.1093/nar/gky508.

15. Anaya, E.U.; Neumann, A. Innate Antifungal Immune Receptor, Dectin-1, Undergoes Ligand-Induced Oligomerization with Highly Structured $\beta$-glucans and at Fungal Cell Contact Sites. Biophys. J. 2020, 118, 244a, doi:10.1016/j.bpj.2019.11.1432.

16. Graus, M.S.; Wester, M.J.; Lowman, D.W.; Williams, D.L.; Kruppa, M.D.; Martinez, C.M.; Young, J.M.; Pappas, H.C.; Lidke, K.A.; Neumann, A.K. Mannan Molecular Substructures Control Nanoscale Glucan Exposure in Candida. Cell Rep. 2018, 24, 2432-2442.e5, doi:10.1016/j.celrep.2018.07.088.

17. Itano, M.S.; Steinhauer, C.; Schmied, J.J.; Forthmann, C.; Liu, P.; Neumann, A.K.; Thompson, N.L.; Tinnefeld, P.; Jacobson, K. Super-Resolution Imaging of C-Type Lectin and Influenza Hemagglutinin Nanodomains on Plasma Membranes Using Blink Microscopy. Biophys. J. 2012, 102, 1534-1542, doi:10.1016/j.bpj.2012.02.022.

18. Manzo, C.; Torreno-Pina, J.A.; Joosten, B.; Reinieren-Beeren, I.; Gualda, E.J.; Loza-Alvarez, P.; Figdor, C.G.; Garcia-Parajo, M.F.; Cambi, A. The Neck Region of the C-type Lectin DC-SIGN Regulates Its Surface Spatiotemporal Organization and Virus-binding Capacity on Antigen-presenting Cells. J. Biol. Chem. 2012, 287, 38946-38955, doi:10.1074/jbc.M112.380121.

19. Rosa, G. de la; Yáñez-Mó, M.; Samaneigo, R.; Serrano-Gómez, D.; MartínezMuñoz, L.; Fernández-Ruiz, E.; Longo, N.; Sánchez-Madrid, F.; Corbí, Á.L.; Sánchez-Mateos, P. Regulated recruitment of DC-SIGN to cell-cell contact regions during zymosan-induced human dendritic cell aggregation. J. Leukoc. Biol. 2005, 77, 699-709, doi:10.1189/j1b.0904529.

20. Itano, M.S.; Neumann, A.K.; Liu, P.; Zhang, F.; Gratton, E.; Parak, W.J.; Thompson, N.L.; Jacobson, K. DC-SIGN and Influenza Hemagglutinin Dynamics in Plasma Membrane Microdomains Are Markedly Different. Biophys. J. 2011, 100, 2662-2670, doi:10.1016/j.bpj.2011.04.044.

21. Liu, P.; Weinreb, V.; Ridilla, M.; Betts, L.; Patel, P.; de Silva, A.M.; Thompson, N.L.; Jacobson, K. Rapid, directed transport of DC-SIGN clusters in the plasma membrane. Sci. Adv. 2017, 3, doi:10.1126/sciadv.aao1616.

22. Kaizuka, Y.; Douglass, A.D.; Varma, R.; Dustin, M.L.; Vale, R.D. Mechanisms for segregating $\mathrm{T}$ cell receptor and adhesion molecules during immunological synapse formation in Jurkat T cells. Proc. Natl. Acad. Sci. 2007, 104, 20296-20301, doi:10.1073/pnas.0710258105.

23. Liu, P.; Wang, X.; Itano, M.S.; Neumann, A.K.; de Silva, A.M.; Jacobson, K.; Thompson, N.L. Low Copy Numbers of DC-SIGN in Cell Membrane Microdomains: Implications for Structure and Function: Quantification of DC-SIGN Copy Numbers in Microdomains. Traffic 2014, 15, 179-196, doi:10.1111/tra.12138.

24. Arana, E.; Vehlow, A.; Harwood, N.E.; Vigorito, E.; Henderson, R.; Turner, M.; Tybulewicz, V.L.J.; Batista, F.D. Activation of the Small GTPase Rac2 via the B Cell Receptor Regulates B Cell Adhesion and Immunological-Synapse Formation. Immunity 2008, 28, 88-99, doi:10.1016/j.immuni.2007.12.003. 
1422

1423

1424

1425

1426

1427

1428

1429

1430

1431

1432

1433

1434

1435

1436

1437

1438

1439

1440

1441

1442

1443

1444

1445

1446

1447

1448

1449

1450

1451
25. Tsourkas, P.K.; Raychaudhuri, S. Modeling of B cell Synapse Formation by Monte Carlo Simulation Shows That Directed Transport of Receptor Molecules Is a Potential Formation Mechanism. Cell. Mol. Bioeng. 2010, 3, 256-268, doi:10.1007/s12195-010-0123-1.

26. Zhang, Y.; Hoppe, A.D.; Swanson, J.A. Coordination of Fc receptor signaling regulates cellular commitment to phagocytosis. Proc. Natl. Acad. Sci. 2010, 107, 19332-19337, doi:10.1073/pnas.1008248107.

27. Geijtenbeek, T.B.H.; Kwon, D.S.; Torensma, R.; van Vliet, S.J.; van Duijnhoven, G.C.F.; Middel, J.; Cornelissen, I.L.M.H.A.; Nottet, H.S.L.M.; KewalRamani, V.N.; Littman, D.R.; et al. DC-SIGN, a Dendritic Cell-Specific HIV-1-Binding Protein that Enhances trans-Infection of T Cells. Cell 2000, 100, 587-597, doi:10.1016/S0092-8674(00)80694-7.

28. van Gisbergen, K.P.J.M.; Sanchez-Hernandez, M.; Geijtenbeek, T.B.H.; van Kooyk, Y. Neutrophils mediate immune modulation of dendritic cells through glycosylation-dependent interactions between Mac-1 and DC-SIGN. J. Exp. Med. 2005, 201, 1281-1292, doi:10.1084/jem.20041276.

29. Lipke, P.N.; Klotz, S.A.; Dufrene, Y.F.; Jackson, D.N.; Garcia-Sherman, M.C. Amyloid-Like $\beta$-Aggregates as Force-Sensitive Switches in Fungal Biofilms and Infections. Microbiol. Mol. Biol. Rev. 2017, 82, e00035-17, doi:10.1128/MMBR.00035-17.

30. Snyder, G.A.; Ford, J.; Torabi-Parizi, P.; Arthos, J.A.; Schuck, P.; Colonna, M.; Sun, P.D. Characterization of DC-SIGN/R Interaction with Human Immunodeficiency Virus Type 1 gp120 and ICAM Molecules Favors the Receptor's Role as an Antigen-Capturing Rather than an Adhesion Receptor. J. Virol. 2005, 79, 4589-4598, doi:10.1128/JVI.79.8.4589-4598.2005.

31. Yokosuka, T.; Sakata-Sogawa, K.; Kobayashi, W.; Hiroshima, M.; HashimotoTane, A.; Tokunaga, M.; Dustin, M.L.; Saito, T. Newly generated T cell receptor microclusters initiate and sustain $\mathrm{T}$ cell activation by recruitment of Zap70 and SLP76. Nat. Immunol. 2005, 6, 1253-1262, doi:10.1038/ni1272. 